\title{
1 An atlas of plant full-length RNA reveals tissue-specific and 2 evolutionarily-conserved regulation of poly(A) tail length
}

4 Jinbu Jia ${ }^{1,2,3 \dagger}$, Wenqin $\mathrm{Lu}^{1,2,3 \dagger}$, Bo Liu ${ }^{1,2,3}$, Yiming Yu${ }^{1,2,3}$, Xianhao Jin ${ }^{1,2,3}$, Yi Shu ${ }^{1,2,3}$,

5 Yanping Long ${ }^{1,2,3}$, and Jixian Zhai ${ }^{1,2,3^{*}}$

7 Affiliations:

$8 \quad{ }^{1}$ Department of Biology, School of Life Sciences, Southern University of Science and

9 Technology, Shenzhen 518055, China;

$10 \quad{ }^{2}$ Institute of Plant and Food Science, Southern University of Science and Technology, Shenzhen

11 518055, China;

$12{ }^{3}$ Key Laboratory of Molecular Design for Plant Cell Factory of Guangdong Higher Education

13 Institutes, Southern University of Science and Technology, Shenzhen 518055, China.

14

$15 \dagger$ These authors contributed equally to this work.

16 * Correspondence: zhaijx@sustech.edu.cn (J.Z.) 


\section{Introductory paragraph}

18 Poly(A) tail is a hallmark of eukaryotic mRNA and its length plays an essential role in regulating

19 mRNA metabolism ${ }^{1-3}$. However, a comprehensive resource for plant poly(A) tail length has yet to

20 be established. Here, we applied a poly(A)-enrichment-free, Nanopore-based method ${ }^{4,5}$ to profile

21 full-length RNA with poly(A) tail information in plants. Our atlas contains over 120 million

22 polyadenylated mRNA molecules from seven different tissues of Arabidopsis, as well as the shoot

23 tissue of maize, soybean and rice. In most tissues, the size of plant poly(A) tails shows peaks at

24 approximately 20 and $45 \mathrm{nt}$, presumably the sizes protected by one and two poly(A) binding

25 proteins ( $\mathrm{PABP}$ ), respectively ${ }^{2,6}$, while the poly(A) tails in pollen exhibit a distinct pattern with

26 strong peaks centered at 55 and $80 \mathrm{nt}$. Moreover, poly(A) tail length is regulated in a gene-specific

27 manner - mRNAs with short half-lives in general have long poly(A) tails, while mRNAs with

28 long half-lives are featured with relatively short poly(A) tails that peak at $\sim 45 \mathrm{nt}$, suggesting that

29 protection of poly(A) tail in this size by PABP is essential for mRNA stability. Across species,

30 poly(A) tails in the nucleus are almost twice as long as in the cytoplasm, implying a conserved

31 rapid shortening process of poly(A) tail occurs before the mRNA is stabilized in cytoplasm. Our

32 comprehensive dataset lays the groundwork for future functional and evolutionary studies on

33 poly(A) tail length regulation in plants.

\section{Main text}

36 A non-template poly(A) tail is the hallmark of eukaryotic mRNA ${ }^{1-3}$ and is vital in promoting

37 translation and protecting mRNAs integrity with the help of cytoplasmic poly(A) binding proteins

38 (PABPC $)^{2,7,8}$. The length of the poly(A) tail is dynamically regulated by poly(A) polymerase and

39 deadenylase, and shorting poly(A) tails to a certain threshold would release PABPC and trigger 
mRNA decay ${ }^{2,3,9-12}$. A growing body of evidence reveals that altering poly(A) tail length plays

41 important role in regulating gene expression ${ }^{2,9,13,14}$. In the last decade, a few high-throughput

42 Illumina-based methods have been developed for genome-wide characterization of poly(A) tail

43 length, including PAL-seq ${ }^{13}$, TAIL-seq ${ }^{15}$, mTAIL-seq $^{16}$, PAT-seq ${ }^{17}$ and TED-seq ${ }^{18}$. Advances in

44 long-read sequencing platforms such as PacBio and Nanopore have also enabled the development

45 of methods that detect full-length mRNA with poly(A) tail information, such as FLAM-seq ${ }^{19}$,

46 PAIso-seq ${ }^{20}$, Nanopore direct RNA sequencing (DRS) $)^{21-23}$, and one developed by our group named

47 FLEP-seq (Full-Length Elongating and Polyadenylated RNA sequencing) ${ }^{4,5}$. However, existing

48 resources on plant poly(A) tails are still limited ${ }^{8,13,21,24-26}$. Therefore, establishing a comprehensive

49 landscape for poly(A) tail length from various tissue types and in different species would greatly

50 facilitate the study of poly(A) regulation in plants.

52 Here, for low-input and cost-efficient detection of poly(A) tails, we further optimized FLEP-seq

53 procedure for studying total RNA and streamlined library construction with the newly released

54 Nanopore PCR-cDNA sequencing kit which uses ligase-free attachment of rapid sequencing

55 adapter to bypass the steps of ds-cDNA repair, A-tailing and adapter ligation — we named this

56 new version of the protocol as FLEP-seq2 (Fig. 1a and Fig. S1). Because FLEP-seq is originally

57 designed to detect nascent RNAs that may or may not have a poly(A) tail, it does not involve any

58 step for selecting poly(A) tail. This turns out to be a unique advantage for unbiased measurement

59 of poly(A) tail length, as all other long-read methods (FLAM-seq, PAIso-seq, and DRS) use oligo-

$60 \mathrm{dT}$ to select poly(A)+ mRNAs, which could disfavor mRNAs with a short poly(A) tail (Fig. 1a).

61 Indeed, compared to published DRS data of the same tissue ${ }^{21}$, FLEP-seq2 detects more transcripts

62 with short poly(A) tail, and the poly(A) length distribution of transcripts shows the highest peak 
63 at 20 nucleotides (nt) (Fig. 1b, upper panel). Despite this slight difference, the overall poly(A)

64 length distributions obtained by FLEP-seq2 and DRS are highly similar (Fig. 1b, upper panel).

65 The median poly(A) length of genes between FLEP-seq2 and DRS ( $r=0.72)$ (Fig. 1b, lower panel,

66 Fig. S2a), as well as between the two biological replicates of FLEP-seq2 $(r=0.87)$ (Fig. S2b), are

67 highly consistent. In addition, FLEP-seq2 has a significant advantage in term of output per

68 Nanopore flow cell compared to DRS — on a regular Nanopore MinION flow cell, one FLEP-

69 seq2 library can yield $\sim 10-20$ million raw reads (Fig. 1a, Fig. 1d), whereas the output of DRS on

70 the same MinION flow cell is only $\sim 1$ million $^{21,22}$. FLEP-seq2 also uses much less input RNA than

71 DRS. DRS requires 500 to 1000 ng polyadenylated RNA ${ }^{21,22}$, while FLEP-seq2 can start with as

72 little as $500 \mathrm{ng}$ total RNA. The full-length information obtained by FLEP-seq2 also enables us to

73 simultaneously track the splicing status, poly(A) site position and poly(A) tail length of each

74 transcript (Fig. 1c). These results demonstrated that FLEP-seq2 is an unbiased, robust, and high-

75 throughput method for measuring poly(A) tail length; therefore, we chose FLEP-seq2 for a

76 comprehensive characterization of poly(A) tail in plants.

78 To establish a comprehensive atlas to investigate the tissue-specificity and evolutionary

79 conservation of poly(A) tail length regulation in plants, we first extracted the total RNA of seven

80 different tissues (seedling, root, shoot, leaf, inflorescence, seed, pollen) of Arabidopsis, as well as

81 the shoot tissues of three important crops: maize, rice, soybean, with two biological replicates for

82 each sample (Fig. 1d). We constructed 20 FLEP-seq2 libraries, each sequenced with a MinION

83 flow cell, which in total yielded $\sim 276$ million raw reads and $\sim 121$ million poly(A)+ reads (Fig. 1d,

84 Table S1). The median poly(A) length of gene is mainly between $50 \mathrm{nt}$ and $100 \mathrm{nt}$ (Table S2),

85 while the poly(A) length distribution of all reads has two prominent peaks, one at $\sim 20 \mathrm{nt}$ and the 
86 other at $\sim 45 \mathrm{nt}$ (Fig. 1 d, Fig. 1e). A peak in $\sim 70 \mathrm{nt}$ can also be observed in some samples, such as

87 soybean and maize (Fig. 1e). These peaks are typically phased with an interval of 25 to $30 \mathrm{nt}$ (Fig.

88 1e), consistent with the footprint of one PABPC protein ${ }^{2,6,27-29}$, suggesting a large portion of

89 mRNAs are protected by PABPC in plants.

91 To investigate if the differences in poly(A) tail length among different genes originated from the

92 nascent RNA, we also analyzed the poly(A) tail length of nascent RNA in the nuclei from

93 Arabidopsis, rice, soybean and maize (Table S3). To our surprise, the poly(A) tail lengths of the

94 nuclear RNA are much longer, mainly at 100-200 nt, with the median length of nuclear RNAs are

95 almost twice the size as to those of total RNAs (Fig. S3), and the median poly(A) tail lengths of

96 genes are also considerably longer than those in total RNAs (Fig. 2a). We previously reported that

97 a large portion of plant nascent transcripts are fully transcribed, polyadenylated, yet incompletely

98 spliced, and these intron-containing nascent RNAs are presumably tethered to the chromatin until

99 they complete splicing ${ }^{5}$. Based on this model, the transcripts with introns detected in total RNAs

100 are mostly the incompletely spliced RNAs in the nuclear. Indeed, we observed that the intron-

101 containing transcripts in total RNA have longer poly(A) tails than the fully spliced ones (Fig. 2b,

102 Fig. 2c, Fig. S4a), and the poly(A) tail lengths of these transcripts with introns from total RNA are

103 similar to those from nuclear RNA (Fig. 2b, Fig. 2d, Fig. S4b). Interestingly, in the nuclei, poly(A)

104 tail lengths of the intron-containing transcripts and fully-spliced transcripts are largely the same

105 (Fig. 2b), suggesting shortening of poly(A) tail is a rapid process that occurs after splicing is 
108 In addition, we found that the poly(A) tail length of about $1 / 5$ of alternatively spliced isoforms

109 (exclude intron retention) are significantly longer than those of the corresponding major isoforms

110 (the most expressed isoforms) in all detected four species (Fig. 2e). Using public Arabidopsis RNA

111 seq data $^{30}$, we found that most of these isoforms, e.g., an alternative 3' splicing site event

112 generating isoform in AT3G01480 (Fig. S5), are upregulated in up frameshift 1 (upf1) upf3 mutant

113 (Fig. 2f), which disrupts the cytoplasmic nonsense-mediated decay (NMD) pathway. This

114 suggested that most of them are the targets of the NMD pathway. NMD is a cytoplasmic mRNA

115 surveillance mechanism which primarily recognizes target RNA during the first round of

116 translation and mediates rapid degradation of their targets ${ }^{31,32}$. Based on this model, for these

117 NMD-targeted isoforms, the detected transcripts/reads should mainly be newly synthesized RNAs

118 which are still in the nucleus and haven't undergone the first round of translation. Consistent with

119 this, these isoforms are enriched in nuclear (Fig. S6), and their poly(A) tail lengths are consistent

120 with those of the transcripts with introns (Fig. S7). All these results indicated that nuclear nascent

121 RNA has a long poly(A) tail and a global deadenylation of mature mRNAs occurs in the cytoplasm

122 of plant cells.

123

124 Transcripts from different genes have poly(A) tails of distinct lengths (Fig. 1d, right panel).

125 However, the poly(A) tails of nuclear nascent RNAs are generally different from those of total

126 RNAs (Fig. 2b), indicating that these intergenic length differences of poly(A) tail in steady-state

127 transcripts should be largely determined by the cytoplasmic deadenylation step. Using the reported

128 genome-wide dataset of mRNA half-lives in Arabidopsis ${ }^{33}$, we found that the poly(A) tails of the

129 most unstable transcripts, i.e., the transcripts from genes with shortest mRNA half-lives, are

130 mainly 70-150 nt, similar to or slightly shorter than those of nuclear RNAs (Fig. 2g, 2h). For these 
131 genes, a large number of transcripts with short poly(A) tails in 10-20 nt are also detected, while

132 the transcripts with poly(A) tails in 20-70 nt are few, implying that they undergo a rapid

133 deadenylating step that shortens the poly(A) in the cytoplasm. On the contrary, the poly(A) tail

134 lengths of the stable transcripts, especially for the most stable transcripts, are distinctly shorter

135 than those of nuclear nascent RNAs, and usually enriched in the range of 20 to 80 nt with phased

136 peaks presumably due to PABPC-binding (Fig. $2 \mathrm{~g}, 2 \mathrm{~h}$ ). These results suggest stable mRNAs

137 initially undergo cytoplasmic deadenylation but are subsequently protected by PABPs against

138 further deadenylating and decay. These results indicated the different poly(A) tail lengths that we

139 observed for different genes could be partly explained by the regulation of differential

140 deadenylation.

142 Next, we investigated whether there is tissue-specific regulation of poly(A) tail length. Although

143 the poly(A) tail length distributions are similar among most tissues, the pattern in pollen and seed

144 are distinct (Fig. 1d, Fig. 3a). The poly(A) tails of transcripts in pollen and seed, especially in

145 pollen, are mainly composed of medium size, with few shortest and longest poly(A) tails (Fig. 1d,

146 Fig. 3a), which is reminiscent of the feature of stable transcripts in other tissues (Fig. 2g). The

147 poly(A) tail distribution of pollen RNAs has three peaks with a $\sim 26$ nt phase interval (Fig. 1e).

148 Moreover, different from other tissues in which the poly(A) tails are enriched at 20-30 nt and 40-

$14960 \mathrm{nt}$, the poly(A) tails of pollen are mainly at 40-60 nt and 70-90 nt (Fig. 1d, Fig. 3a), suggesting

150 that the transcripts in pollen are potentially bound and protected by more PABPs. The poly(A) tails

151 of mRNAs with short half-lives in seedling also enriched at 40-90 nt and has two peaks at 40-60

152 nt and 70-90 nt in pollen (Fig. S8a, S8b), implying that many transcripts which are rapidly

153 degraded in other tissues are also protected in pollen. Similar to pollen, poly(A) tail distribution in 
154 seed also exhibits longer tails, although the pattern is less obvious than in pollen (Fig. 1d, Fig. 1e,

155 Fig. 3a). These results indicated a stronger protection by PABPs in pollen and seed, consistent

156 with the previous reports that the mRNAs in pollen are usually stable ${ }^{34}$ and many mRNAs are

157 stored in pollen and seed for the germination of pollen and seed, respectively ${ }^{35,36}$.

159 Besides pollen and seed which are distinctly different from other tissues in poly(A) tail length

160 distribution, the correlation coefficients of the median poly(A) tails of genes among the other five

161 tissues are also lower than the correlation coefficients between two biological replicates from the

162 same tissue (Fig. S9a). The heatmap plot of the median poly(A) tail length of genes also showed

163 similar results (Fig. S9b). These results suggested that the poly(A) tails of many genes are tissue-

164 specifically regulated. Consistent with this finding, a large number of genes (range between 250

165 and 1665) showing significantly differentially regulated poly(A) tails were identified in each pair

166 of tissues, compared to only few (8 to 57) differential genes identified from the random data

167 generated by shuffling the samples of two compared tissues (Fig. S9c). For example, the poly(A)

168 tails of AT5G16470.1, AT1G51200.1, AT5G65480.1 are enriched in 10-50 nt length in most

169 tissues, and the poly(A) tail of AT2G01100.1 are enriched in 100-200 nt length, but all of them

170 are enriched in 50-100 nt length in pollen (Fig. 3b). The poly(A) tail of AT1G08830.1 and

171 AT1G09070.1 are differentially shorter in leaf and inflorescence than those in seedling, shoot and

172 root (Fig. 3b). And the poly(A) tails of AT3G57450.1 are shorter in leaf than those in seedling,

173 shoot, root and inflorescence (Fig. 3b).

175 The poly(A) length distributions of different species are quite similar, but also show some

176 differences (Fig. 1d, Fig. 3c). Compared to Arabidopsis shoot, rice shoot has more transcripts with 
177 an extremely short tail (Fig. 1d), and more genes have a peak at 10-20 nt in poly(A) distribution

178 (Fig. 3c), and thus the median poly(A) tail lengths of genes are shorter (Fig. 1d). In contrast, maize

179 and soybean shoot, especially for soybean shoot, has fewer transcripts with an extremely shorter

180 tail, but has more transcripts with a longer tail (Fig. 1d, Fig. 3c), and showed a higher peak in 60-

$18180 \mathrm{nt}$ (Fig. 1d, Fig. 1e), which might represent transcripts protected by three PABPs. Despite these

182 differences, the poly(A) tail lengths of homologous genes are significantly correlated among

183 different species ( $r: 0.49$ to 0.58 ) (Fig. 3d). Take transcription factors as examples, the poly(A)

184 tails of SQUAMOSA PROMOTER BINDING PROTEIN-LIKE 1 (SPL1) and SPL12, which act

185 redundantly in thermotolerance at the reproductive stage ${ }^{37}$, has a broader distribution in length;

186 ETHYLENE INSENSITIVE 3 and its closest homolog EIN3-LIKE1 (EIL1), two key regulators in

187 ethylene signal transduction pathway, have more poly(A) tails in 20-100 nt; the poly(A) tails of

188 METHYLENE BLUE SENSITIVITY 2 (MBS2), a mediator of singlet oxygen responses ${ }^{38}$, enriched

189 at 20-50 nt; the poly(A) tails of ETHYLENE RESPONSE FACTOR 4 (ERF4) / ERF8 / ERF9 have

190 two peaks in length, one at 50-100 nt and the other at 10-20 nt; and the poly(A) tails of $A U X I N$

191 RESPONSE FACTOR 10 (ARF10) / ARF16 are mainly higher than 100-nt (Fig. 3e). These results

192 suggested that the poly(A) tail lengths of orthologous genes are relatively conserved among

193 different plant species, and thus might be selected under evolutionary pressure.

195 Our data showed that the poly(A) tail lengths can be different among different genes but highly

196 correlated among different tissues for the same gene (Fig. S9a, $r \geqslant 0.8$ for most tissue pairs

197 except for pollen and seed), and are evolutionarily conserved in different species. These results

198 indicate that they are tightly controlled in gene-specific ways in plants, thus may reflect their

199 critical roles in gene regulation. The poly(A) tails of total RNA are significantly shorter than those 
of nuclear RNA, indicating that the poly(A) lengths of steady-state mRNA are largely dependent on the cytoplasmic deadenylation process. If deadenylation rate is uniform for a given gene, the poly(A) tail length distribution should be broad and flat, such as the pattern of SPL1/SPL12 genes shown in Fig. 3e. However, the poly(A) tail of many plant transcripts, especially for stable RNAs,

204 have peaks at 20-60 nt, indicating that they first undergo rapid deadenylation and then be protected 205 when poly(A) tails become 20-60 nt. This is consistent with the dual roles of PABPC and the biphasic deadenylation model reported in animals and yeast - PABPC can stimulate the

207 deadenylation of long poly(A) tails via binding to the deadenylase complexes but blocking 208 precocious decay ${ }^{1,2,27,28}$. However, the homologs of the executor which initially trim the long 209 poly(A) tails of nascent RNA in this model, PAN2/PAN3 ${ }^{2,27}$, although conserved in animals and 210 yeast, haven't been identified in the Arabidopsis genome ${ }^{39}$, implying other deadenylase complexes 211 may replace them to perform the initial trimming. Besides, the poly(A) tail of some transcripts, 212 especially for those with short half-lives, are longer and rarely in 20-60 nt, but usually are also 213 enriched in 10-20 nt (Fig 2g, 3a, 3c), a size that may be short enough to loosen or lost their 214 association with PABP and has been reported to prefer for being uridylated and decay ${ }^{24,28,40}$, 215 implying an accelerated deadenylation from long poly(A) tail to extremely short, consistent with 216 the canonical mRNA decay model that the poly(A) tail is first shortened to $10-12$ nt before further 217 decay from both 5'-3' and 3'-5' direction ${ }^{2}$. These results suggested that there could be multiple 218 modes of deadenylation in plants, which are gene-specifically regulated, highlighting the 219 importance of profiling poly(A) lengths of different genes.

221 It has been reported that the poly(A) tail lengths are globally changed in specific animal systems, 222 such as the oocyte-to-embryo development stage, a model system to study the function of poly(A) 
223 tail ${ }^{1,2,13,16}$. Here, our data highlights that pollen and seed show distinct poly(A) tail length

224 distribution among plant tissues. The poly(A) tails of pollen and seed RNAs are enriched in 40-

$22590 \mathrm{nt}$, thus might be protected by more PABPC proteins and serve the purpose of storing mRNA

226 in these two tissues ${ }^{34-36}$. Arabidopsis genome contains eight PABPC genes $^{8,41}$. Previous reports ${ }^{8,41}$

227 and the public RNA-seq dataset ${ }^{42}$ reveal that $P A B 2$ and its two closest homologs, $P A B 4$ and $P A B 8$,

228 are highly expressed in a wide range of tissues, while $P A B 3, P A B 5, P A B 6$ and $P A B 7$ are expressed

229 in pollen (Fig. S10). Further study on these pollen-specifically expressed PABs will help explore

230 the unique mechanism of poly(A) length regulation in pollen and its roles in stabilizing mRNA.

231 Finally, the comprehensive landscape of poly(A) tails from various tissues and species will provide

232 an important resource to explore the dynamic regulation of poly(A) length and its roles in

233 controlling gene expression in plants. 


\section{Methods}

\section{Plant materials and RNA isolation}

237 Arabidopsis ecotype Col-0, soybean cultivar Wm82, rice cultivar Nipponbare and maize cultivar 238 B73 are used in this study. For Arabidopsis, plants were grown at $22^{\circ} \mathrm{C}$ with $16 \mathrm{~h}$ of light per 24 239 hours. Arabidopsis seedlings, shoots and roots were harvested after growing on 1/2 MS plates for

24012 days. Arabidopsis leaves were harvested after growing in soil for 30 days, and Arabidopsis

241 inflorescences were harvested after flowering. For rice, soybean and maize, plants were grown at

$24228^{\circ} \mathrm{C}$ with 16 hours of light per 24 hours, and 14-day-old shoots were harvested. The nuclear

243 fractions were separated as described ${ }^{4,5}$, and the total RNA and nuclear RNA were extracted using

244 RNAprep Pure Plant Plus Kit (Polysaccharides \& Polyphenolics-rich, TIANGEN, DP441)

245 according to the manufacturer's instructions.

\section{Library preparation and Nanopore sequencing}

248 FLEP-seq2 libraries were prepared from 500-3000 ng input RNA as previously described ${ }^{4,5}$ with

249 some improvement. In brief, the ribosomal RNA (rRNA) was removed using pan-plant riboPOOLs

250 probes (siTOOLs Biotech) and Dynabeads Myone Streptavidin C1 (Thermo Fisher, \#65001)

251 according to the manufacturer's instruction (siTOOLs Biotech, two-step depletion method).

252 rRNA-depleted RNA was purified by RNA Clean \& Concentrator-5 kit (ZYMO, R1013) and then

253 ligated to 50 pmol 3' adapter (5' - rAppCTGTAGGCACCATCAAT $-\mathrm{NH}_{2}-3^{\prime}$ ) in a $20 \mu \mathrm{l}$

254 reaction containing 1X T4 RNA Ligase Reaction Buffer, 25\% PEG8000, 40U Murine RNase

255 Inhibitor (Vazyme, R301-03) and 20U T4 RNA Ligase 2, truncated K227Q (NEB, M0242) for 10

$256 \mathrm{~h}$ at $16^{\circ} \mathrm{C}$. The product was cleaned up using RNA Clean \& Concentrator-5 kit (ZYMO, R1013)

257 and added into $20 \mu \mathrm{l}$ of reverse transcription and strand-switching reaction containing $100 \mathrm{nM}$ 
custom primer (5' - phos/ ACTTGCCTGTCGCTCTATCTTCATTGATGGTGCCTACAG - 3'), $500 \mu \mathrm{M}$ dNTPs, 1X RT Buffer, 40U Murine RNase Inhibitor (Vazyme, R301-03), $1 \mu \mathrm{M}$ Strand-

261 (Thermo Fisher, EP0752), and then incubated at $90 \mathrm{~min} 42^{\circ} \mathrm{C} ; 10$ cycles of $\left(2 \mathrm{~min}\right.$ at $50^{\circ} \mathrm{C} ; 2 \mathrm{~min}$

262 at $42^{\circ} \mathrm{C}$ ); and $5 \mathrm{~min}$ at $85^{\circ} \mathrm{C}$, in a thermal cycler. cDNA libraries were amplified with PrimeSTAR

263 GXL DNA polymerase (TaKaRa, R050A) for 10-16 cycles. To minimize PCR bias, PCR cycle

264 number optimization was performed as previously described ${ }^{4,5}$. After PCR, $1 \mu$ l of Exonuclease I

265 (NEB, M0293) was added to the reaction mixture and incubated the reaction at $37^{\circ} \mathrm{C}$ for $15 \mathrm{~min}$,

266 followed by $80^{\circ} \mathrm{C}$ for 15 minutes. The products were cleaned up with $0.8 \mathrm{X}$ Ampure XP beads

267 (Beckman, A63880). For the Nanopore sequencing, 100 fmol amplified cDNA was loaded onto

268 an R9.4 Flow Cell (Oxford Nanopore Technologies) using Sequence-specific cDNA-PCR

269 Sequencing kit (Nanopore, SQK-PCS109) and sequenced on a MinION device for $\sim 48$ hours.

$271 \quad$ Nanopore data processing

272 The Nanopore data pre-processing was performed using FLEP-seq pipeline

273 (https://github.com/ZhaiLab-SUSTech/FLEPSeq) as previously described with minor

274 modification about adapter sequence ${ }^{4,5}$. Briefly, the raw Nanopore signals were converted to base

275 sequences by Guppy (v4.0.11, download from Oxford Nanopore Community) with the default

276 parameters (--c dna_r9.4.1_450bps_hac.cfg). The reads with a mean quality score of more than 7

277 were mapped to genome sequence using Minimap2 (v.2.17-r943-dirty) ${ }^{43}$ with the following 278 parameters: -ax splice --secondary $=$ no $-\mathrm{G}$ 12000. The used genome and annotation versions of

279 Arabidopsis, soybean, rice, maize were ARAPROT11 (https://www.arabidopsis.org/),

280 Wm82.gnm4.ann1.T8TQ (https://soybase.org/), MSU7 (http://rice.uga.edu), B73 RefGen_v5 
281 (https://maizegdb.org), respectively. The reads mapped to rDNA, mitochondria and chloroplast

282 genomes were removed by filter_rRNA_bam.py in FLEP-seq pipeline. The 3' adapters were

283 identified by adapterFinder.py with parameter: --mode 1. The 5' and 3' adapter sequences of

284 FLEP-seq2 are different from those of FLEP-seq, and are TTTCTGTTGGTGCTGATATTGCT

285 and ACTTGCCTGTCGCTCTATCTTCATTGATGGTGCCTACAG, respectively. This

286 modification has been integrated into the new version of adapterFinder.py (set '--mode 1'

287 parameter for FLEP-seq2, and '--mode 0' or default for FLEP-seq) in FLEP-seq pipeline. Then,

288 poly(A) tail identification and length estimation were performed by PolyACaller.py and the

289 splicing status of intron was extracted by extract_read_info.py. The transcripts/reads with a

290 predicated poly(A) tail score equal to or more than 10 were identified as polyadenylated transcripts

291 and used for further analysis. Only the reads spanning all introns were used for the analysis of the

292 fully-spliced and intron-containing transcripts, and the intron with a mapping ratio of at least $80 \%$

293 in a read was identified as unspliced intron. To identify orthologs among different species, the

294 protein sequences of all four species were exported to OrthoFinder software ${ }^{44}$ with default

295 parameters.

297 Identification of alternative splicing isoform from Nanopore data

298 We first extracted splicing junctions from reads mapped to a given gene to identify high credible

299 splicing events. Due to the higher base error ratio, the alignment quality of nanopore reads near

300 the splice site is relatively poor, and the end positions of splicing junctions might be wrong. Thus,

301 if both ends of multiple detected splicing junctions are close (within $10 \mathrm{nt}$ ), they might be generated

302 from wrong alignments, and only the splicing junction with the most supported reads was remained.

303 The set of overlapping splicing junctions represents a group of alternative splicing (AS) events. 
304 For a given splicing junction $(J)$, the percent spliced in (PSI) value of $J$ equal to $n 1 /[n 1+n 2] ; n 1$ :

305 the number of reads specifically supported $J ; n 2$ : the number of reads specifically supported the

306 splicing junctions overlapping with $J$. The splicing junction with less than 20 supported reads or

307 with a PSI value lower than $2 \%$ were removed. Multiple introns of one gene might undergo AS,

308 and thus one gene could contain multiple AS groups. Therefore, we second joined these AS

309 junctions to AS isoforms based on the supporting reads. For a given gene, the reads spanning all

310 AS groups were extracted. The splicing statuses of all AS junctions in each read were extracted,

311 and the intron-containing transcripts/reads were removed. Each remained read was explicitly

312 derived from one kind of isoform, and all supported AS junction combinations/isoforms as well as

313 the number of supported reads were calculated. The isoforms with more than 20 supported reads

314 were identified as highly creditable isoforms, and the poly(A) tails of the reads specifically

315 supporting them were used for the poly(A) tail length analysis of isoforms

\section{Isoform quantification}

318 For a given gene, unique representative splicing junction of each isoform was extracted.

319 Considering that the sequencing depth of the 3' end of a gene is usually higher, if one isoform

320 contains two or more unique representative splicing junctions, only the most downstream one was

321 used. For each sample, the number of reads supported the unique representative splicing junction

322 of each isoform $\left(n_{j}\right)$ was extracted, and the minor/major isoform ratio was calculated by

$323 n_{j_{-} \text {minor }} / n_{j_{-} \text {major }}$. 
326 For comparison of two groups (e.g., tissue $\mathrm{S}$ and tissue $\mathrm{R}$, or major isoforms $\mathrm{S}$ and minor isoforms

327 R) with biological replicates (e.g., S1, S2, R1 and R2), for each gene, the poly(A) tail lengths of

328 gene-derived reads detected in each sample $\left(X_{S 1}, X_{S 2}, X_{R 1}, \mathrm{X}_{\mathrm{R} 2}\right)$ were extracted, and the medians of

329 poly(A) tail lengths of each sample $\left(M_{S 1}, M_{S 2}, M_{R 1}, M_{R 2}\right)$ were calculated. The difference factor

330 of median $(d m)$ was calculated by $d m=\max (\min (0, d m 1), \min (0, d m 2)) ; d m 1=\min \left(\mathrm{M}_{\mathrm{s} 1}, \mathrm{M}_{\mathrm{s} 2}\right)-$

$331 \max \left(\mathrm{M}_{\mathrm{R} 1}, \mathrm{M}_{\mathrm{R} 2}\right) ; \mathrm{dm} 2=\min \left(\mathrm{M}_{\mathrm{R} 1}, \mathrm{M}_{\mathrm{R} 2}\right)-\max \left(\mathrm{M}_{\mathrm{S} 1}, \mathrm{M}_{\mathrm{S} 2}\right) . X_{S 1}$ and $X_{S 2}$ were merged to $X_{S}$, and $X_{R 1}$

332 and $X_{R 2}$ were merged to $X_{R}$. Then, for each gene, a two-sided Mann-Whitney $\mathrm{U}$ test between $X_{S}$

333 and $X_{R}$ was performed, and the p-values of all genes were adjusted by Benjamini/Hochberg FDR

334 (False Discovery Rate) method. The genes with an adjusted p-value less than 0.05 and a value $d m$

335 more than 20 were identified as the genes showing differential poly(A) tail length between samples.

Data Availability

338 The raw sequencing data generated in this study were deposited in China National Center for

339 Bioinformation with accession PRJCA007575 and in NCBI with accession PRJNA788163

340 (https://dataview.ncbi.nlm.nih.gov/object/PRJNA788163?reviewer=hlvulfu2lo8062gc5fhuuiupf6

341 for reviewer link). The poly(A) tail lengths of reads from each gene in each library were recorded

342 in China National Center for Bioinformation with accession OMIX881. The median poly(A)

343 lengths of genes in each library were recorded in Table S2.

345 Correspondence and requests for materials should be addressed to Jixian Zhai 346 (zhaijx@sustech.edu.cn). 
349 The group of J.Z. is supported by the National Key R\&D Program of China Grant 350 (2019YFA0903903); the Program for Guangdong Introducing Innovative and Entrepreneurial 351 Teams (2016ZT06S172); the Shenzhen Sci-Tech Fund (KYTDPT20181011104005); the Key

352 Laboratory of Molecular Design for Plant Cell Factory of Guangdong Higher Education Institutes

353 (2019KSYS006); and the Stable Support Plan Program of Shenzhen Natural Science Fund Grant

354 (20200925153345004). J.J. is supported by the National Natural Science Foundation of China

355 (32100444); and the Shenzhen Fundamental Research Program (20210317103146009).

357 Author Contributions

358 J.Z., J.J. and W.L. designed the experiments. W.L., J.J., B.L., X.J., Y.S. and Y.L. performed the

359 experiments. J.J., W.L. and Y.Y. analyzed the data. J.Z. oversaw the study. J.J., W.L. and J.Z.

360 wrote the manuscript, and all authors revised the manuscript.

\section{Competing interests}

363 The authors declare no competing interests. 


\section{$364 \quad$ Figure Legends}

365 Figure 1. An atlas of plant poly(A)-tail lengths measured by FLEP-seq2. a, The schematic

366 diagram of FLEP-seq2 and Direct RNA sequencing (DRS). RT: reverse transcription. b, The

367 distribution of global poly(A) tail lengths of transcripts/reads (upper panel, $1 \mathrm{nt}$ bin) and the median

368 poly(A) tail length of genes (bottom panel, $5 \mathrm{nt}$ bin, only genes with at least 20 reads were used)

369 measured by FLEP-seq and DRS. c, An example of reads aligned to the AT1G08830 gene in a

370 FLEP-seq2 library (seedling replicate 1). Only polyadenylated reads were shown. d, The

371 distribution of global poly(A) tail lengths of transcripts/reads (left panel) and the median poly(A)

372 tail length of genes (right panel) in different tissues and different species. In the analysis of median

373 poly(A) tail length of genes, only genes with at least 20 reads were used. Rep \#1: biological

374 replicate 1; rep \#2: biological replicate 2. e, The peaks of global poly(A) tail length distribution in

375 representative samples.

377 Figure 2. Nuclear Poly(A) tails are longer than tails in cytoplasm. a, The distribution of median

378 poly(A) tail lengths of nuclear and total RNA from different plant species (5 nt bin). Only genes

379 with at least 20 detected reads in both nuclear and total RNA libraries were used. b. The

380 distribution of median poly(A) tail lengths of fully spliced transcripts and intron-containing

381 transcripts (with introns) from nuclear and total RNA in different plant species (5 nt bin). Only

382 genes with at least 20 fully spliced reads and 20 intron-containing reads in both nuclear and total

383 RNA libraries were used. c, The comparison of median poly(A) tail lengths between fully spliced

384 transcripts and intron-containing (with introns) transcripts in Arabidopsis seedling samples. Only

385 the transcripts/reads spanning all annotated introns were used, and only genes with at least 20 fully

386 spliced reads and 20 intron-containing reads are used. $\mathbf{d}$, The comparison of median poly(A) tail 
lengths of intron-containing transcripts between nuclear and total RNA in Arabidopsis seedling.

388 Only the transcripts/reads spanning all annotated introns were used, and only genes with at least

38920 intron-containing transcripts in both nuclear and total RNA libraries were used. e, The

390 comparison of median poly(A) tail lengths between minor and major alternative-splicing (exclude

391 intron-retention) generating isoforms. Only isoforms with at least 20 reads were used. For each

392 gene, the isoform with the highest expression was designed as major isoforms. All other isoforms

393 were designed as minor isoforms and were compared to the major isoform. The numbers of

394 isoforms showing no differential, longer, and shorter poly(A) tails were separated with ":" and

395 labeled above each figure. f, Boxplot showing the distribution of $\Delta$ minor isoform ratio

396 (minor/[minor+major]) of mutants. The sum of the number of minor and major isoform reads in

397 each sample is required to be more than 10. g, Heatmap plot showing the poly(A) tail length

398 distribution of genes with different half-lives. The mRNA half-life data of seedling was reported

399 in previous paper (Szabo et al., 2020). Each row in the plot represents the poly(A) tail length

400 distribution of one gene. Only genes with at least 50 reads were used. $\mathbf{h}$, The bulk poly(A) tail

401 length distribution of genes with different mRNA half-lives in Arabidopsis. N: gene number.

Figure 3. Tissue-specific and evolutionarily-conserved regulation of poly(A) tail length in

404 plants. a, Heatmap plot showing the poly(A) tail length distribution of genes in different tissues.

405 Only genes with at least 50 reads were used. b, Examples of genes showing differential poly(A)

406 tail length distribution in different tissues. Inflo.: Inflorescence. r1: biological replicate 1; r2:

407 biological replicate 2. c, Heatmap plot showing the poly(A) tail length distribution of genes in

408 different species. Only genes with at least 50 reads were used. d, The correlation of the median

409 poly(A) tail length of orthologous gene pair in different species. Only genes with more than 50 
bioRxiv preprint doi: https://doi.org/10.1101/2022.0121.477033; this version posted January 22, 2022. The copyright holder for this preprint (which was not certified by peer review) is the author/funder, who has granted bioRxiv a license to display the preprint in perpetuity. It is made available under aCC-BY-NC-ND 4.0 International license.

410 reads were used. The Pearson's $r$ values were labeled above each figure. e, Examples of the poly(A)

411 tail length distributions of homologous genes among different species. N: gene number.

412 


\section{References}

4141 Nicholson, A. L. \& Pasquinelli, A. E. Tales of Detailed Poly(A) Tails. Trends Cell Biol. 29, 191-200, doi:10.1016/j.tcb.2018.11.002 (2019).

4162 Passmore, L. A. \& Coller, J. Roles of mRNA poly(A) tails in regulation of eukaryotic gene 417 expression. Nat. Rev. Mol. Cell Biol., doi:10.1038/s41580-021-00417-y (2021).

4183 Eckmann, C. R., Rammelt, C. \& Wahle, E. Control of poly(A) tail length. Wiley Interdiscip. 419 Rev. RNA 2, 348-361, doi:10.1002/wrna.56 (2011).

4204 Long, Y., Jia, J., Mo, W., Jin, X. \& Zhai, J. FLEP-seq: simultaneous detection of RNA polymerase II position, splicing status, polyadenylation site and poly(A) tail length at genome-wide scale by single-molecule nascent RNA sequencing Nat. Protoc. 16, 43554381, doi:10.1038/s41596-021-00581-7 (2021).

5 Jia, J. et al. Post-transcriptional splicing of nascent RNA contributes to widespread intron retention in plants. Nat. Plants 6, 780-788 doi:10.1038/s41477-020-0688-1 (2020).

6 Lima, S. A. et al. Short poly(A) tails are a conserved feature of highly expressed genes. Nat. Struct. Mol. Biol. 24, 1057-1063, doi:10.1038/nsmb.3499 (2017).

7 Kühn, U. \& Wahle, E. Structure and function of poly(A) binding proteins. Biochim. Biophys. Acta. 1678, 67-84, doi:10.1016/j.bbaexp.2004.03.008 (2004). in enhancing translational efficiency. Genome Biol. 20, 189, doi:10.1186/s13059-0191799-8 (2019). mRNA control. Nat. Rev. Mol. Cell Biol. 9, 337-344, doi:10.1038/nrm2370 (2008).

$11 \mathrm{Vi}$, S. L. et al. Target specificity among canonical nuclear poly(A) polymerases in plants modulates organ growth and pathogen response. Proc. Natl. Acad. Sci. US A 110, 1399413999, doi:10.1073/pnas.1303967110 (2013).

13 Subtelny, A. O., Eichhorn, S. W., Chen, G. R., Sive, H. \& Bartel, D. P. Poly(A)-tail profiling reveals an embryonic switch in translational control. Nature 508, 66-71 doi:10.1038/nature13007 (2014). the cytoplasmic polyadenylation complex. Mol. Cell 47, 253-266, doi:10.1016/j.molcel.2012.05.016 (2012). 
44915 Chang, H., Lim, J., Ha, M. \& Kim, V. N. TAIL-seq: Genome-wide Determination of

450 Poly(A) Tail Length and 3' End Modifications. Mol. Cell 53, 1044-1052 doi:10.1016/j.molcel.2014.02.007 (2014).

45216 Lim, J., Lee, M., Son, A., Chang, H. \& Kim, V. N. mTAIL-seq reveals dynamic poly(A) tail regulation in oocyte-to-embryo development. Genes Dev. 30, 1671-1682 doi:10.1101/gad.284802.116 (2016).

17 Harrison, P. F. et al. PAT-seq: a method to study the integration of $3^{\prime}$-UTR dynamics with gene expression in the eukaryotic transcriptome. $R N A$ 21, 1502-1510, doi:10.1261/rna.048355.114 (2015).

18 Woo, Y. M. et al. TED-Seq Identifies the Dynamics of Poly(A) Length during ER Stress. Cell Rep. 24, 3630-3641, doi:10.1016/j.celrep.2018.08.084 (2018).

19 Legnini, I., Alles, J., Karaiskos, N., Ayoub, S. \& Rajewsky, N. FLAM-seq: full-length mRNA sequencing reveals principles of poly(A) tail length control. Nat. Methods 16, 879886 doi:10.1038/s41592-019-0503-y (2019).

20 Liu, Y., Nie, H., Liu, H. \& Lu, F. Poly(A) inclusive RNA isoform sequencing (PAIso-seq) reveals wide-spread non-adenosine residues within RNA poly(A) tails. Nat. Commun. 10, 5292 doi:10.1038/s41467-019-13228-9 (2019).

21 Parker, M. T. et al. Nanopore direct RNA sequencing maps the complexity of Arabidopsis mRNA processing and m6A modification. eLife 9, e49658 doi:10.7554/eLife.49658 (2020).

Workman, R. E. et al. Nanopore native RNA sequencing of a human poly(A) transcriptome. Nat. Methods 16, 1297-1305, doi:10.1038/s41592-019-0617-2 (2019).

23 Parker, M. T. et al. Widespread premature transcription termination of Arabidopsis thaliana NLR genes by the spen protein FPA. Elife 10, doi:10.7554/eLife.65537 (2021).

Scheer, H. et al. The TUTase URT1 connects decapping activators and prevents the accumulation of excessively deadenylated mRNAs to avoid siRNA biogenesis. Nat. Commun. 12, 1298, doi:10.1038/s41467-021-21382-2 (2021). Modulated by mRNA Poly(A) Tail Length. Front. Plant Sci. 11, doi:10.3389/fpls.2020.01255 (2020).

27 Schafer, I. B. et al. Molecular Basis for poly(A) RNP Architecture and Recognition by the

28 Yi, H. et al. PABP Cooperates with the CCR4-NOT Complex to Promote mRNA

29 Webster, M. W. et al. mRNA Deadenylation Is Coupled to Translation Rates by the doi:10.1016/j.molcel.2018.05.033 (2018). 
30 Drechsel, G. et al. Nonsense-mediated decay of alternative precursor mRNA splicing variants is a major determinant of the Arabidopsis steady state transcriptome. Plant Cell 25, 3726-3742, doi:10.1105/tpc.113.115485 (2013).

49131 Kervestin, S. \& Jacobson, A. NMD: a multifaceted response to premature translational termination. Nat. Rev. Mol. Cell Biol. 13, 700-712, doi:10.1038/nrm3454 (2012).

32 Kurosaki, T., Popp, M. W. \& Maquat, L. E. Quality and quantity control of gene expression by nonsense-mediated mRNA decay. Nat. Rev. Mol. Cell Biol. 20, 406-420, doi:10.1038/s41580-019-0126-2 (2019).

33 Szabo, E. X. et al. Metabolic Labeling of RNAs Uncovers Hidden Features and Dynamics of the Arabidopsis Transcriptome. Plant Cell 32, 871-887 doi:10.1105/tpc.19.00214 (2020).

34 Ylstra, B. \& McCormick, S. Analysis of mRNA stabilities during pollen development and in BY2 cells. Plant J. 20, 101-108, doi:10.1046/j.1365-313x.1999.00580.x (1999).

35 Bai, B. et al. Seed-Stored mRNAs that Are Specifically Associated to Monosomes Are Translationally Regulated during Germination. Plant Physiol. 182, 378-392, doi:10.1104/pp.19.00644 (2020).

Hao, H., Li, Y., Hu, Y. \& Lin, J. Inhibition of RNA and protein synthesis in pollen tube development of Pinus bungeana by actinomycin D and cycloheximide. New Phytol. 165, 721-729, doi:10.1111/j.1469-8137.2004.01290.x (2005).

Chao, L. M. et al. Arabidopsis Transcription Factors SPL1 and SPL12 Confer Plant Thermotolerance at Reproductive Stage. Mol. Plant 10, 735-748, doi:10.1016/j.molp.2017.03.010 (2017).

Shao, N., Duan, G. Y. \& Bock, R. A mediator of singlet oxygen responses in Chlamydomonas reinhardtii and Arabidopsis identified by a luciferase-based genetic screen in algal cells. Plant Cell 25, 4209-4226, doi:10.1105/tpc.113.117390 (2013). A Dynamic Triumvirate Controlling Cytoplasmic mRNA Fate and Function. Plant Physiol. 176, 254-269, doi:10.1104/pp.17.01468 (2018).

$4 \mathrm{Yu}, \mathrm{S} . \& \mathrm{Kim}, \mathrm{V}$. N. A tale of non-canonical tails: gene regulation by post-transcriptional RNA tailing. Nat. Rev. Mol. Cell Biol. 21, 542-556, doi:10.1038/s41580-020-0246-8 (2020).

41 Belostotsky, D. A. Unexpected Complexity of Poly(A)-Binding Protein Gene Families in Flowering Plants: Three Conserved Lineages That Are at Least 200 Million Years Old and Possible Auto- and Cross-Regulation. Genetics 163, 311-319, doi:10.1093/genetics/163.1.311 (2003).

2 Zhang, H. et al. A Comprehensive Online Database for Exploring approximately 20,000 Public Arabidopsis RNA-Seq Libraries. Mol. Plant 13, 1231-1233, doi:10.1016/j.molp.2020.08.001 (2020). 
52643 Li, H. Minimap2: pairwise alignment for nucleotide sequences. Bioinformatics 34, 3094-

527 3100, doi:10.1093/bioinformatics/bty191 (2018).

$52844 \quad$ Emms, D. M. \& Kelly, S. OrthoFinder: phylogenetic orthology inference for comparative 529 genomics. Genome Biol. 20, 238, doi:10.1186/s13059-019-1832-y (2019). 
bioRxiv preprint doi: https://doi.org/10.1101/2022.01.21.477033; this version posted January 22, 2022. The copyright holder for this preprint (which was not certified by peer review) is the author'funder, who has granted bioRxiv a license to display the preprint in perpetuity. It is made

FLEP-seq2

Total RNA

$\downarrow$ rRNA depletion

$\downarrow$ 3' adapter ligation

Template switch RT \&

Full-length cDNA PCR

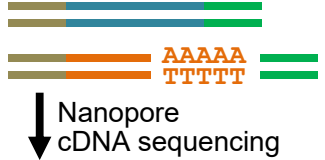

10-20 Million raw reads

(per MinION flow cell)
Direct RNA sequencing

Total RNA

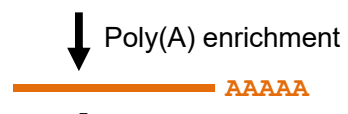

Oligo-dT adapter annealing \& ligation

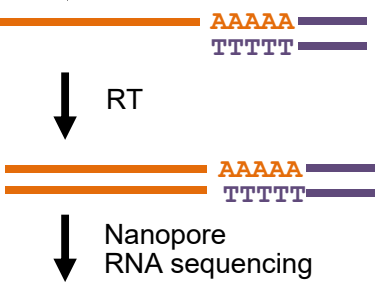

$\sim 1$ Million raw reads

(per MinION flow cell)

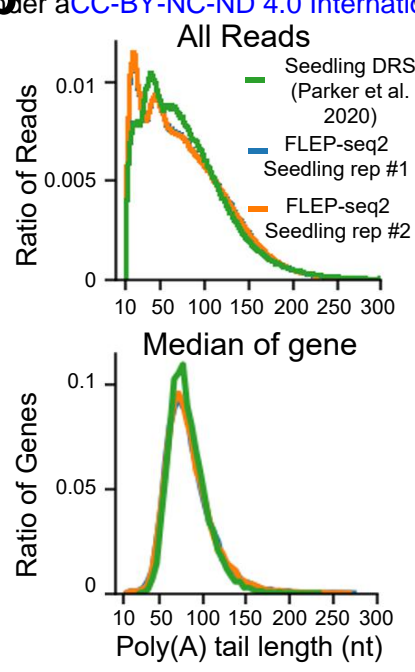

\begin{abstract}
alicense.
\end{abstract}

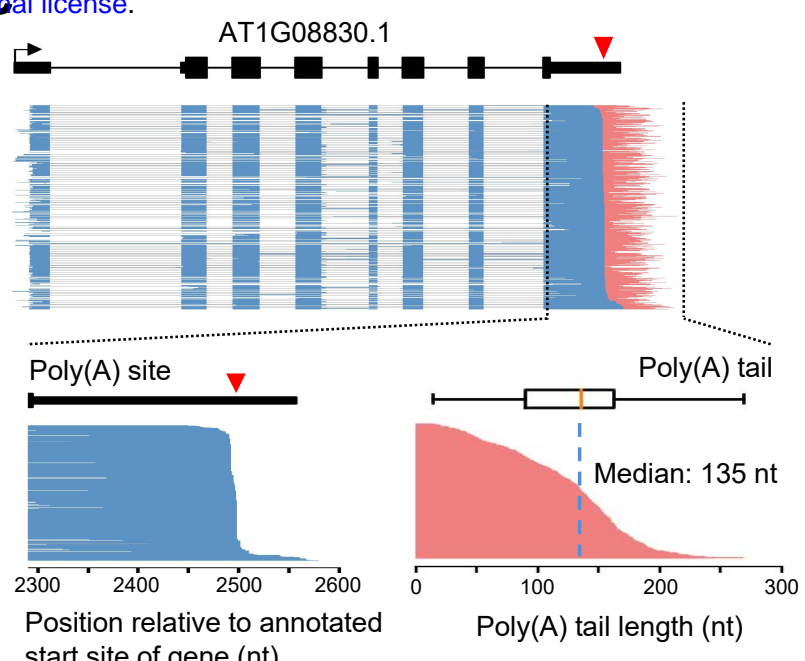

Position relative to annotated start site of gene (nt) d

$\begin{array}{cc}\text { Raw } & \text { Poly(A)+ } \\ \text { Reads } & \text { Reads }\end{array}$

Seedling

Pollen

Inflore-

scence

$y$

Seed

0

rep \#2

Leaf $\$ 0$

rep \#1 $\quad$ 14,964,292 $5,401,251$

rep \#2 $\quad 17,485,397 \quad 5,544,194$

Root s.m. rep\#1 7,018,476 4,463,545

rep \#2 $\quad 11,493,524 \quad 7,254,178$

Shoot<smiles>c1ccc2ccccc2c1</smiles>

rep \#2 13,377,346 $4,561,62$

$\frac{\stackrel{D}{N}}{\sqrt{D}}$

$\frac{\Phi}{ㅁ ㅡ ㅁ ~}$

ஸे

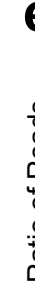
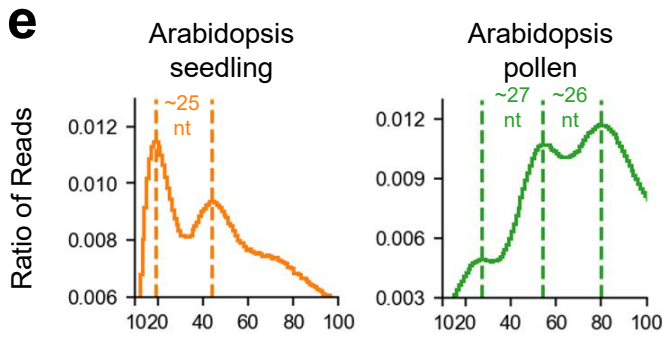

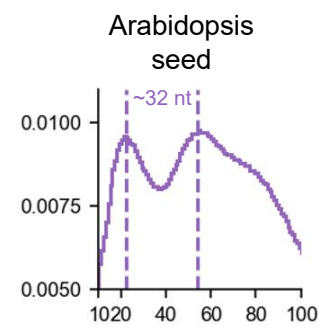

Poly(A) Length of Reads
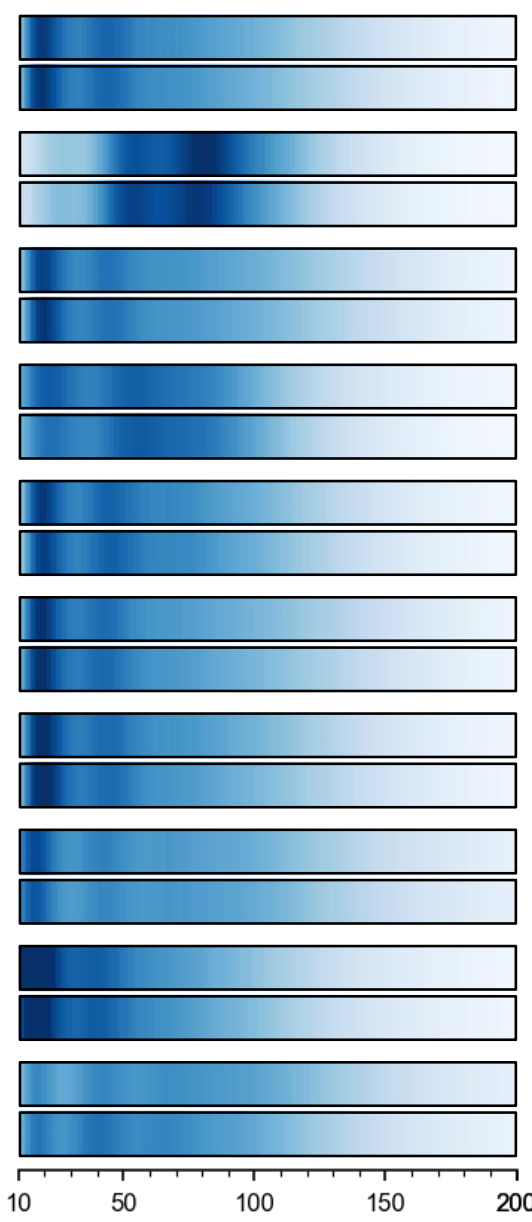

Arabidopsis

Soybean

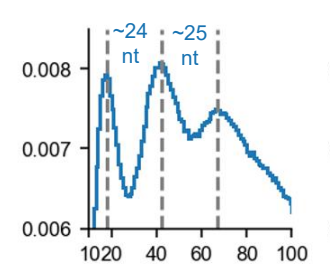

Median Poly(A) Length of Genes
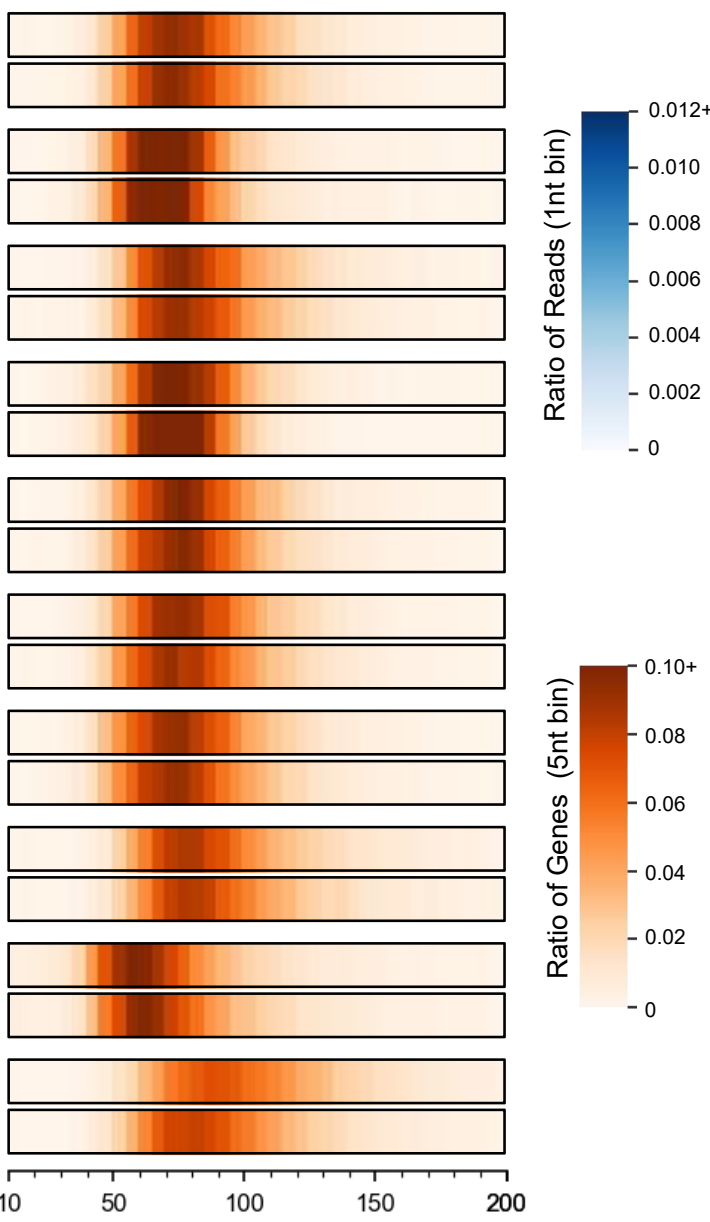

Maize
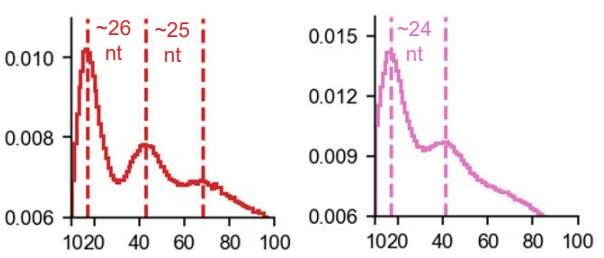

Figure 1. An atlas of plant poly(A)-tail lengths measured by FLEP-seq2. a, The schematic diagram of FLEP-seq2 and Direct RNA sequencing (DRS). RT: reverse transcription. $\mathbf{b}$, The distribution of global poly(A) tail lengths of transcripts/reads (upper panel, $1 \mathrm{nt}$ bin) and the median poly(A) tail length of genes (bottom panel, 5 nt bin, only genes with at least 20 reads were used) measured by FLEP-seq and DRS. c, An example of reads aligned to the AT1G08830 gene in a FLEP-seq2 library (seedling replicate 1). Only polyadenylated reads were shown. d, The distribution of global poly(A) tail lengths of transcripts/reads (left panel) and the median poly(A) tail length of genes (right panel) in different tissues and different species. In the analysis of median poly(A) tail length of genes, only genes with at least 20 reads were used. Rep \#1: biological replicate 1; rep \#2: biological replicate 2. e, The peaks of global poly(A) tail length distribution in representative samples. 
bioRxiv preprint doi: https://doi.org/10.1101/2022.01.21.477033; this version posted January 22, 2022. The copyright holder for this preprint (which was not certified by peer review) is the author/funder, who has granted bioRxiv a license to display the preprint in perpetuity. It is made available under aCC-BY-NC-ND 4.0 International license.

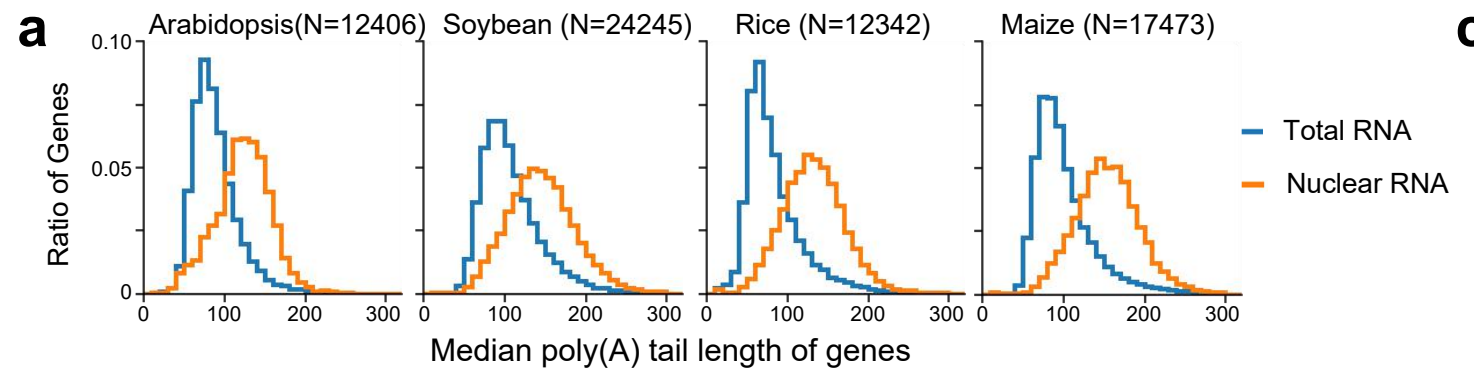

b

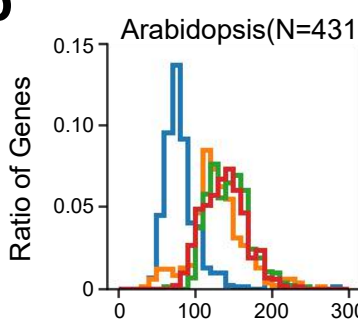

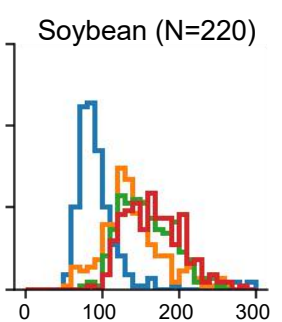

Median poly $(\mathrm{A})$ tail length of genes e

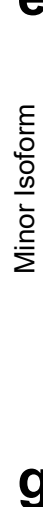

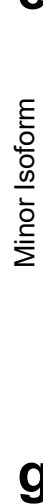

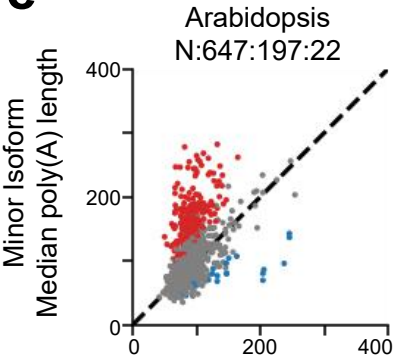

궁

Major Isoform Median poly(A) tail length

Rice

Soybean N:766:151:19

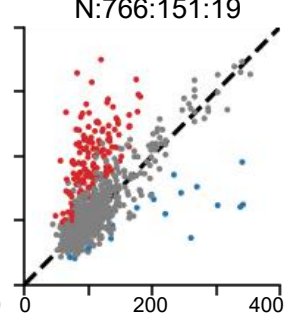

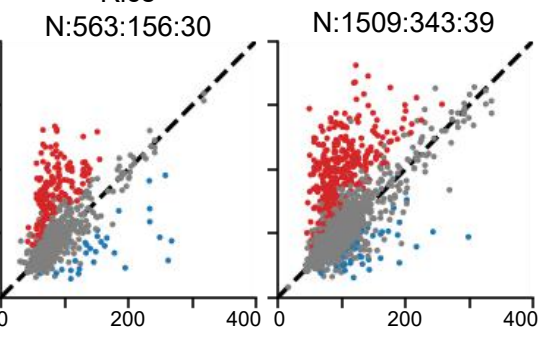
Half-life $>4$ all three replicates $(\mathrm{N}=584)$

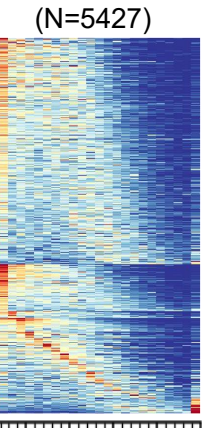

$2<=$ Half-life $<4$ $(\mathrm{N}=4162)$

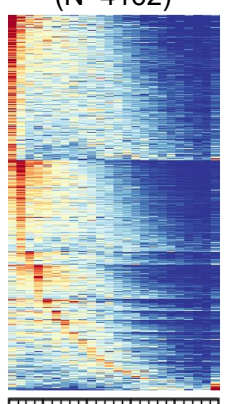

Half-life $>4$ in

Maize $(\mathrm{N}=348)$

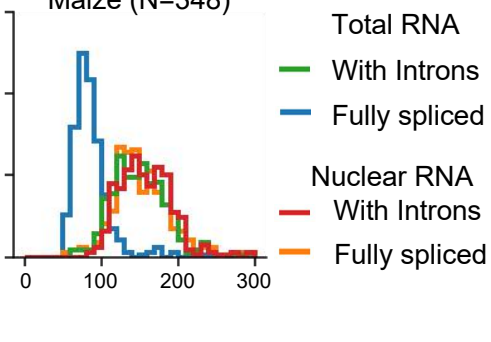

$(\mathrm{N}=117)$

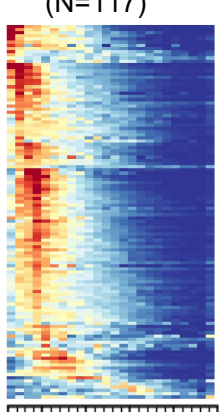

$\mathrm{N}: \bullet: \bullet: \bullet$

- No diff

- Minor

longer

- Major longer

Ratio

(10nt bin)

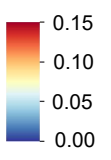

Poly(A)
C

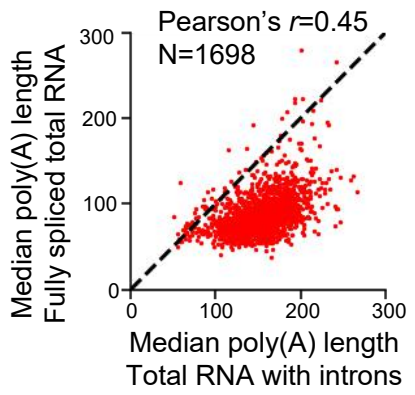

d

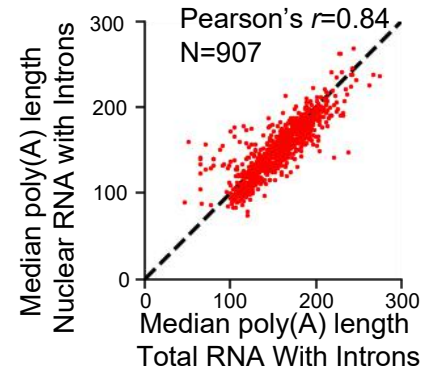

$\mathrm{f}$ h
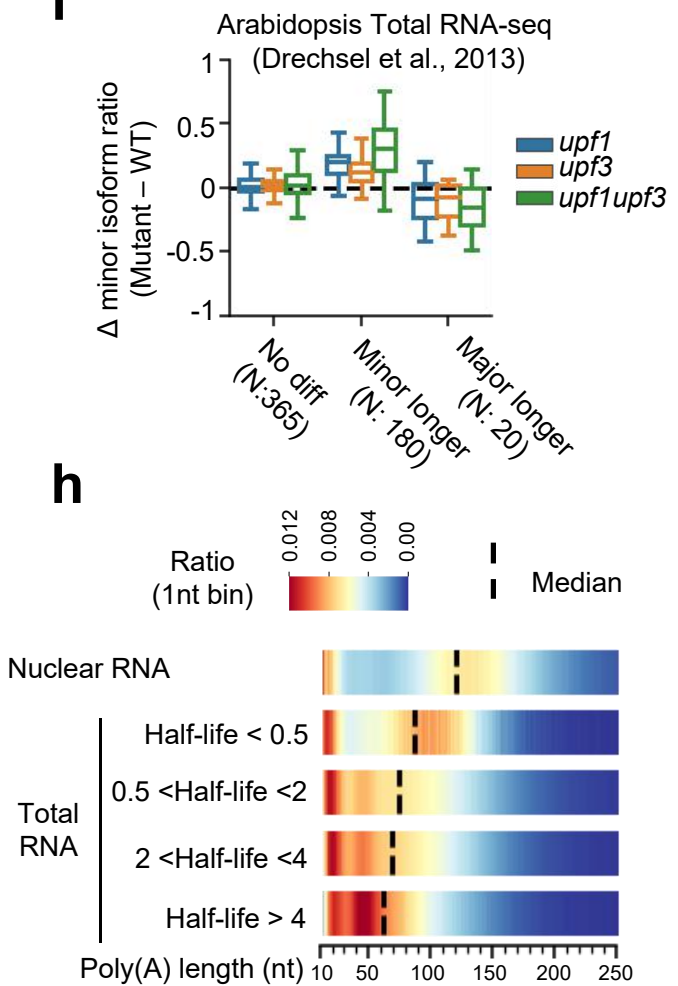

Figure 2. Nuclear Poly(A) tails are longer than tails in cytoplasm. a, The distribution of median poly(A) tail lengths of nuclear and total RNA from different plant species ( $5 \mathrm{nt}$ bin). Only genes with at least 20 detected reads in both nuclear and total RNA libraries were used. b, The distribution of median poly(A) tail lengths of fully spliced transcripts and intron-containing transcripts (with introns) from nuclear and total RNA in different plant species (5 nt bin). Only genes with at least 20 fully spliced reads and 20 intron-containing reads in both nuclear and total RNA libraries were used. c, The comparison of median poly(A) tail lengths between fully spliced transcripts and intron-containing (with introns) transcripts in Arabidopsis seedling samples. Only the transcripts/reads spanning all annotated introns were used, and only genes with at least 20 fully spliced reads and 20 intron-containing reads are used. $\mathbf{d}$, The comparison of median poly(A) tail lengths of intron-containing transcripts between nuclear and total RNA in Arabidopsis seedling. Only the transcripts/reads spanning all annotated introns were used, and only genes with at least 20 introncontaining transcripts in both nuclear and total RNA libraries were used. e, The comparison of median poly(A) tail lengths between minor and major alternative-splicing (exclude intron-retention) generating isoforms. Only isoforms with at least 20 reads were used. For each gene, the isoform with the highest expression was designed as major isoforms. All other isoforms were designed as minor isoforms and were compared to the major isoform. The numbers of isoforms showing no differential, longer, and shorter poly $(\mathrm{A})$ tails were separated with ":" and labeled above each figure. f, Boxplot showing the distribution of $\Delta$ minor isoform ratio (minor/[minor+major]) of mutants. The sum of the number of minor and major isoform reads in each sample is required to be more than 10. g, Heatmap plot showing the poly(A) tail length distribution of genes with different half-lives. The mRNA half-life data of seedling was reported in previous paper (Szabo et al., 2020). Each row in the plot represents the poly(A) tail length distribution of one gene. Only genes with at least 50 reads were used. $\mathbf{h}$, The bulk poly(A) tail length distribution of genes with different $\mathrm{mRNA}$ half-lives in Arabidopsis. N: gene number. 
bioRxiv preprint doi: https://doi.org/10.1101/2022.01.21.477033; this version posted January 22, 2022. The copyright holder for this preprint (which was not certified by peer review) is the author/funder, who has granted bioRxiv a license to display the preprint in perpetuity. It is made available under aCC-BY-NC-ND 4.0 International license.

\section{a $\quad \begin{gathered}\text { Seedling } \\ (\mathrm{N}=13116)\end{gathered}$}
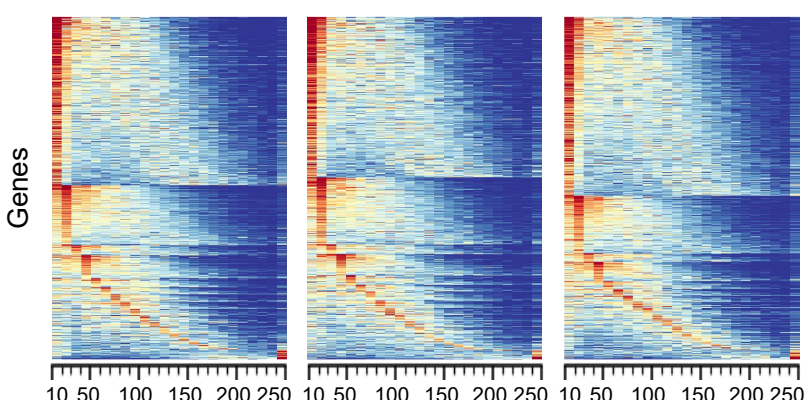

Root

$(\mathrm{N}=13675)$
Leaf $(\mathrm{N}=10653)$

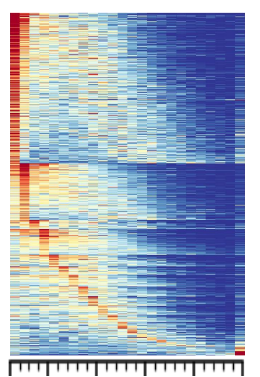

Inflorescence $(\mathrm{N}=14689)$

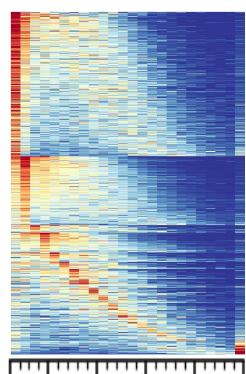

Seed $(\mathrm{N}=9348)$

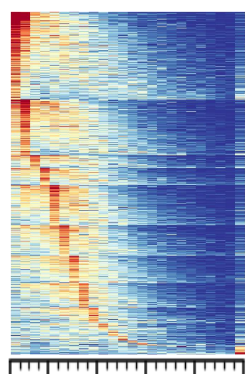

Pollen $(\mathrm{N}=5796)$

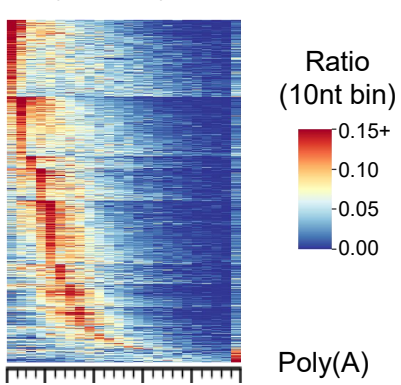

$105010015020025010501001502002501050100150200250105010015020025010501001502002501050 \quad 1001502002501050 \quad 100150200250 \quad$ length (nt)

b

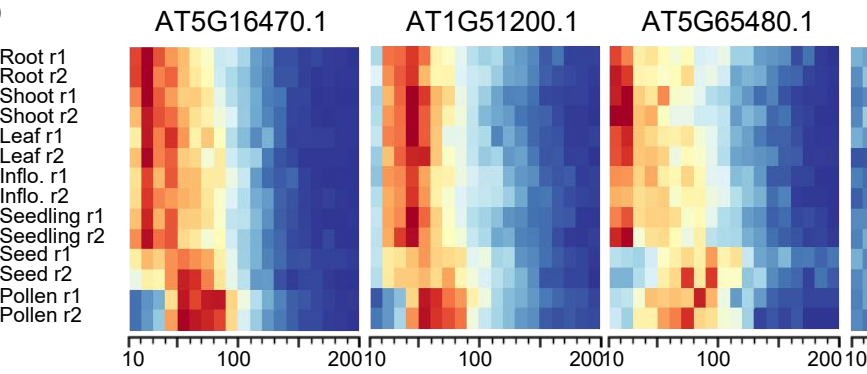

AT2G01100.1

AT1G08830.1

AT1G09070.1

AT3G57450.1
C

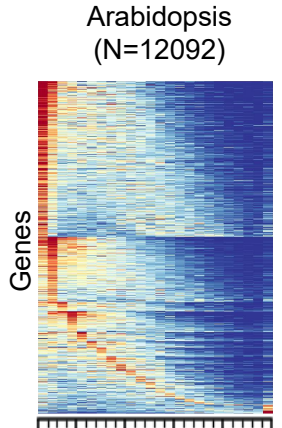

1050100150200250
Soybean
$(\mathrm{N}=21725)$

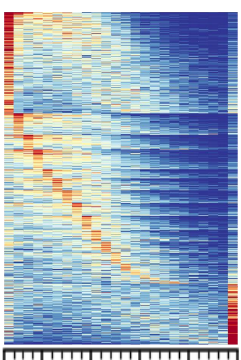

Rice $(\mathrm{N}=11989)$

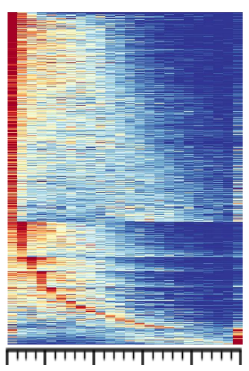

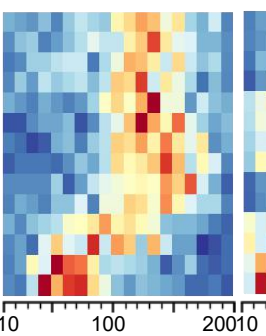
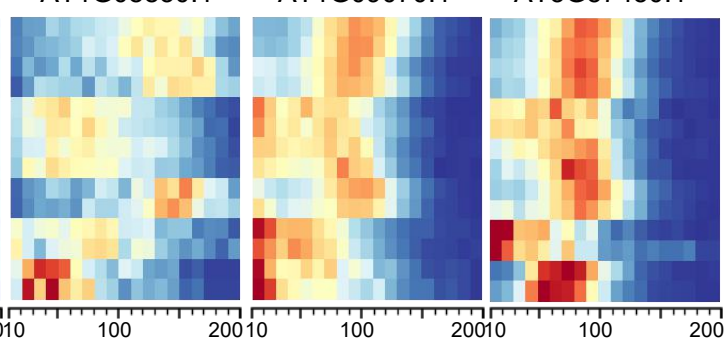

Ratio

(10nt bin)

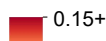

$-0.10$

$-0.05$

0.00

Poly(A) length (nt)

d
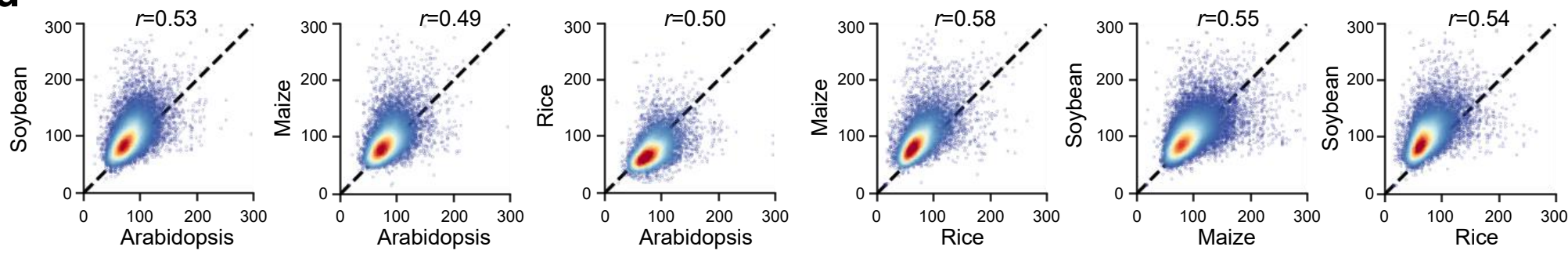

Figure 3. Tissue-specific and evolutionarily-conserved regulation of poly(A) tail length in plants. a, Heatmap plot showing the poly(A) tail length distribution of genes in different tissues. Only genes with at least 50 reads were used. $\mathbf{b}$, Examples of genes showing differential poly $(A)$ tail length distribution in different tissues. Inflo.: Inflorescence. r1: biological replicate 1; r2: biological replicate 2. c, Heatmap plot showing the poly(A) tail length distribution of genes in different species. Only genes with at least 50 reads were used. $\mathbf{d}$, The correlation of the median poly $(\mathrm{A})$ tail length of orthologous gene pair in different species. Only genes with more than 50 reads were used. The Pearson's $r$ values were labeled above each figure. e, Examples of the poly $(\mathrm{A})$ tail length distributions of homologous genes among different species. N: gene number. 
bioRxiv preprint doi: https://doi.org/10.1101/2022.01.21.477033; this version posted January 22, 2022. The copyright holder for this preprint (which was not certified by peer review) is the author/funder, who has granted bioRxiv a license to display the preprint in perpetuity. It is made available under aCC-BY-NC-ND 4.0 International license.

Takara SMARTer PCR cDNA Synthesis Kit

Nanopore DNA ligation sequencing kit (SQK-LSK109)
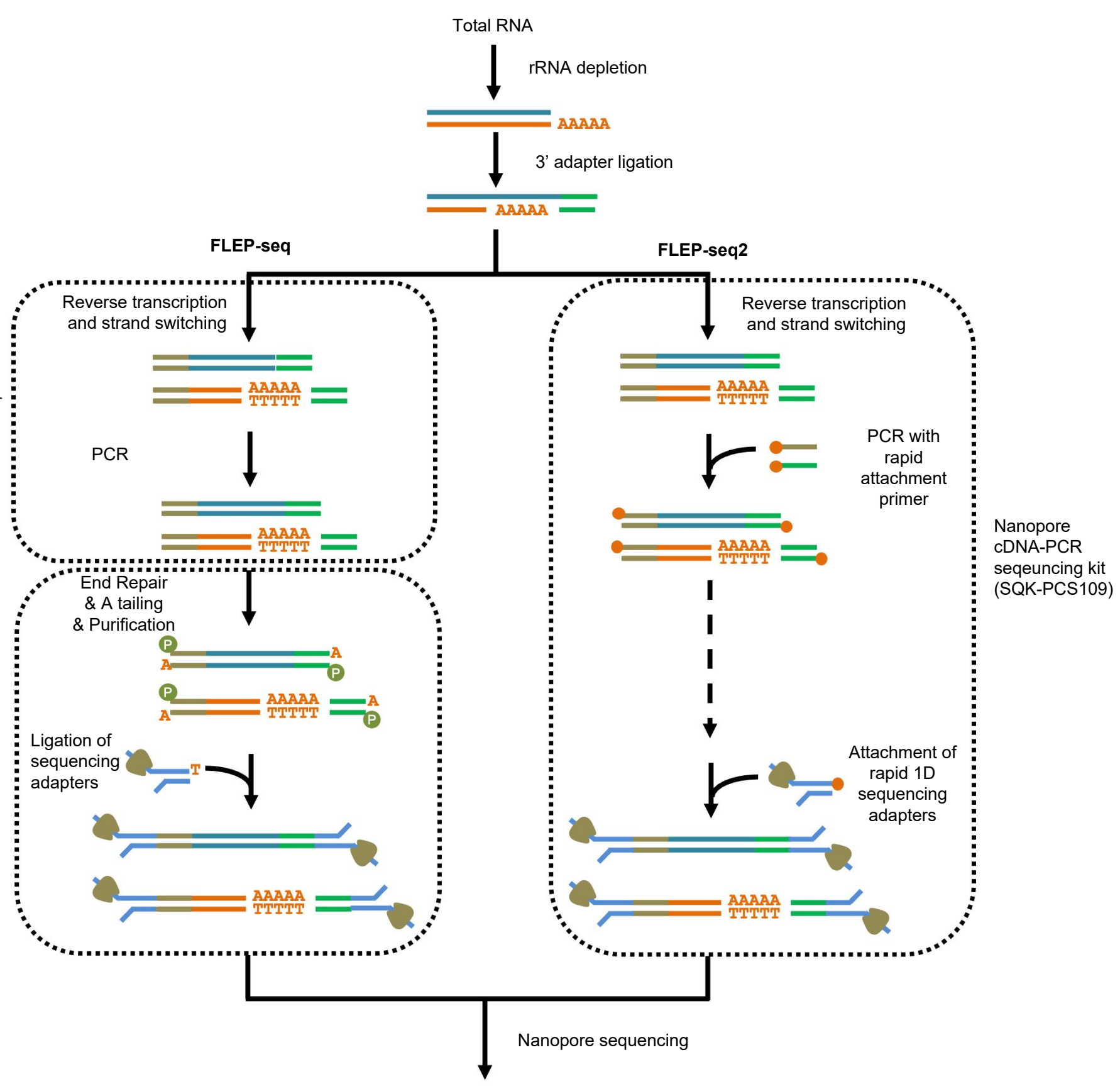

10-20 Million raw reads

(per MinION flow cell)

Supplemental Figure 1. The schematic diagram of FLEP-seq and FLEP-seq2 
bioRxiv preprint doi: https://doi.org/10.1101/2022.01.21.477033; this version posted January 22, 2022. The copyright holder for this preprint (which was not certified by peer review) is the author/funder, who has granted bioRxiv a license to display the preprint in perpetuity. It is made available under aCC-BY-NC-ND 4.0 International license.

a

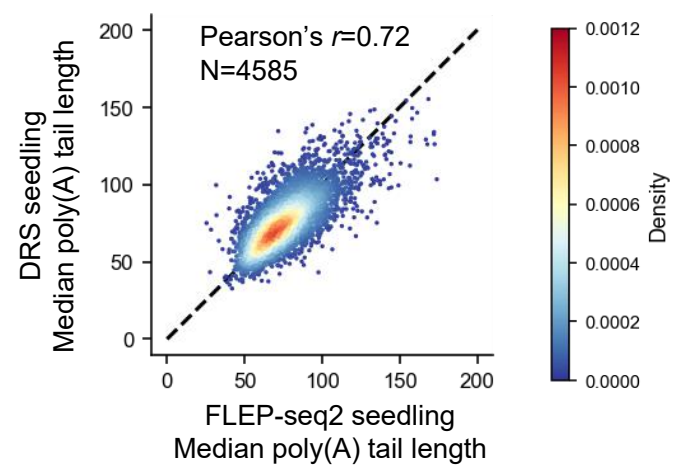

b

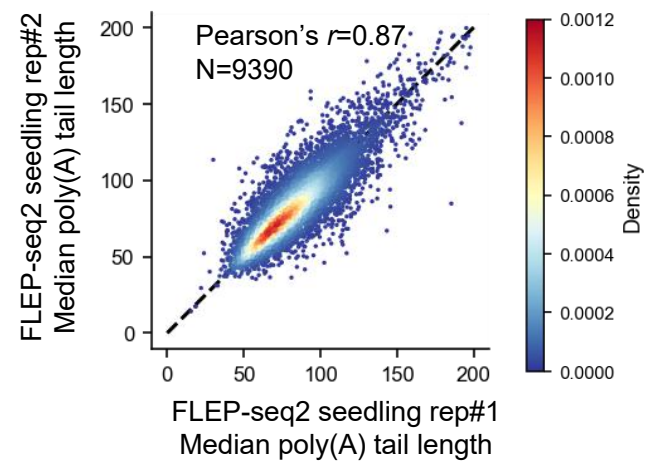

Supplemental Figure 2. The measured median poly(A) lengths of genes are consistent between FLEP-seq2 and DRS dataset and between different biological replicates of FLEP-seq2. a, The correlation of the median poly $(A)$ tail lengths measured by FLEP-seq2 and Direct RNA sequencing (DRS). Only genes with at least 50 reads in both data set were used. $\mathbf{b}$, The correlation of the median poly $(\mathrm{A})$ tail lengths measured by FLEP-seq2 in two different biological replicates. Only genes with at least 50 reads in both samples were used. 
bioRxiv preprint doi: https://doi.org/10.1101/2022.01.21.477033; this version posted January 22, 2022. The copyright holder for this preprint (which was not certified by peer review) is the author/funder, who has granted bioRxiv a license to display the preprint in perpetuity. It is made available under aCC-BY-NC-ND 4.0 International license.

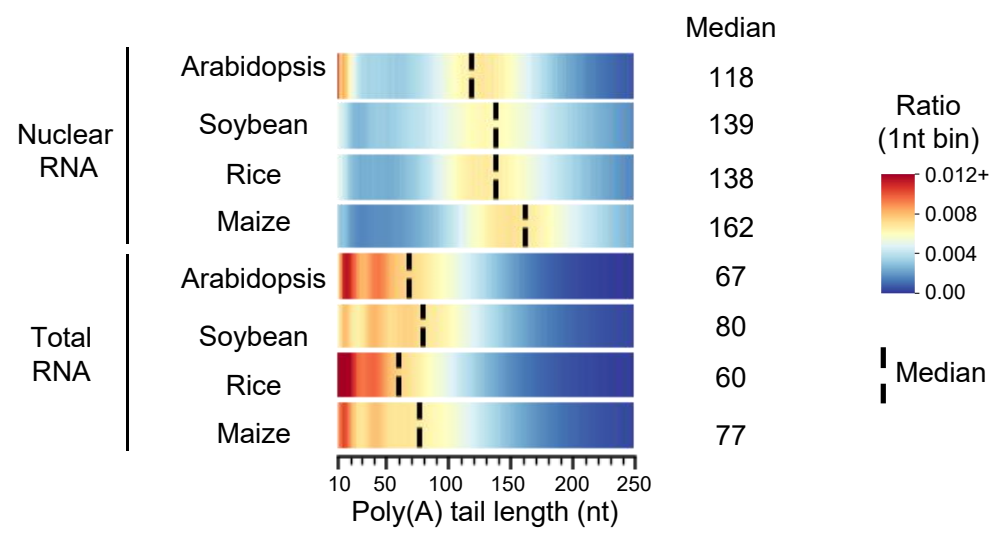

Supplemental Figure 3. The poly(A) tail length distribution of total RNA and nuclear RNA in different species 
bioRxiv preprint doi: https://doi.org/10.1101/2022.01.21.477033; this version posted January 22, 2022. The copyright holder for this preprint (which was not certified by peer review) is the author/funder, who has granted bioRxiv a license to display the preprint in perpetuity. It is made available under aCC-BY-NC-ND 4.0 International license.
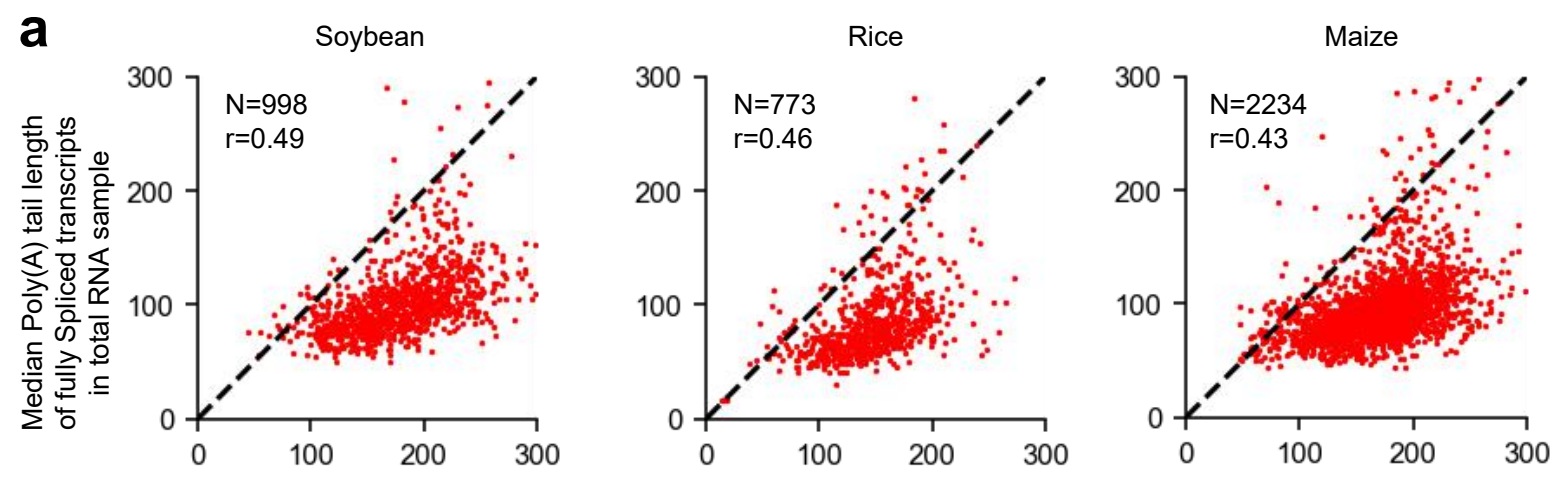

Median poly(A) tail length of intron-containing transcripts in total RNA sample
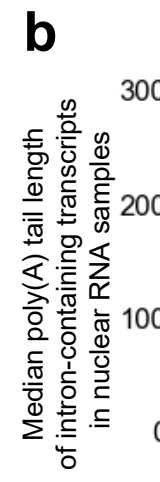
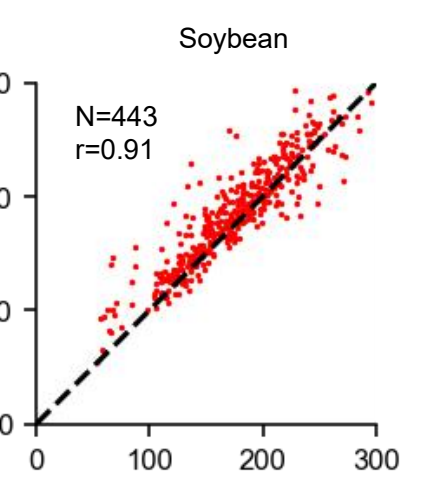

Median poly
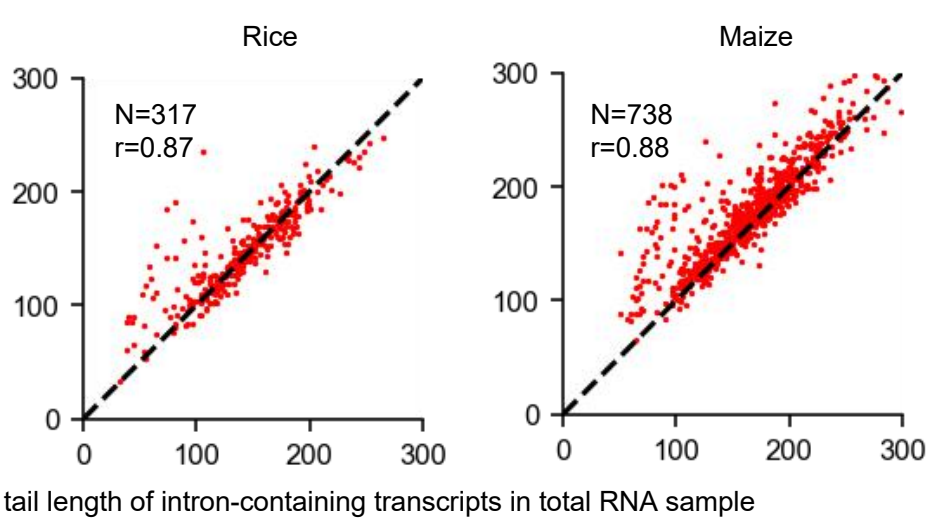

Supplemental Figure 4. Comparison of the poly(A) tail length of intron-containing transcripts and fully-spliced transcripts. a, The comparison of median poly(A) tail lengths between fully spliced transcripts and intron-containing (with introns) transcripts in total RNA samples. Only transcripts spanning all annotated introns were used, and only genes with at least 20 fully spliced reads and 20 intron-containing reads were used. $\mathbf{b}$. The comparison of median poly(A) tail lengths of intron-containing transcripts between nuclear and total RNA samples in different species. Only the transcripts spanning all annotated introns were used, and only genes with at least 20 intron-containing transcripts in both nuclear and total RNA libraries were used. N: gene number. The Pearson's $r$ values were labeled upper each figure. 
bioRxiv preprint doi: https://doi.org/10.1101/2022.0121.477033. this version posted January 22, 2022. The copyright holder for this preprint (which was not certified by peer review) is the author/funder, who has granted bioRxiv a license to display the preprint in perpetuity. It is made available under aCC-BY-NC-ND 4.0 International license.

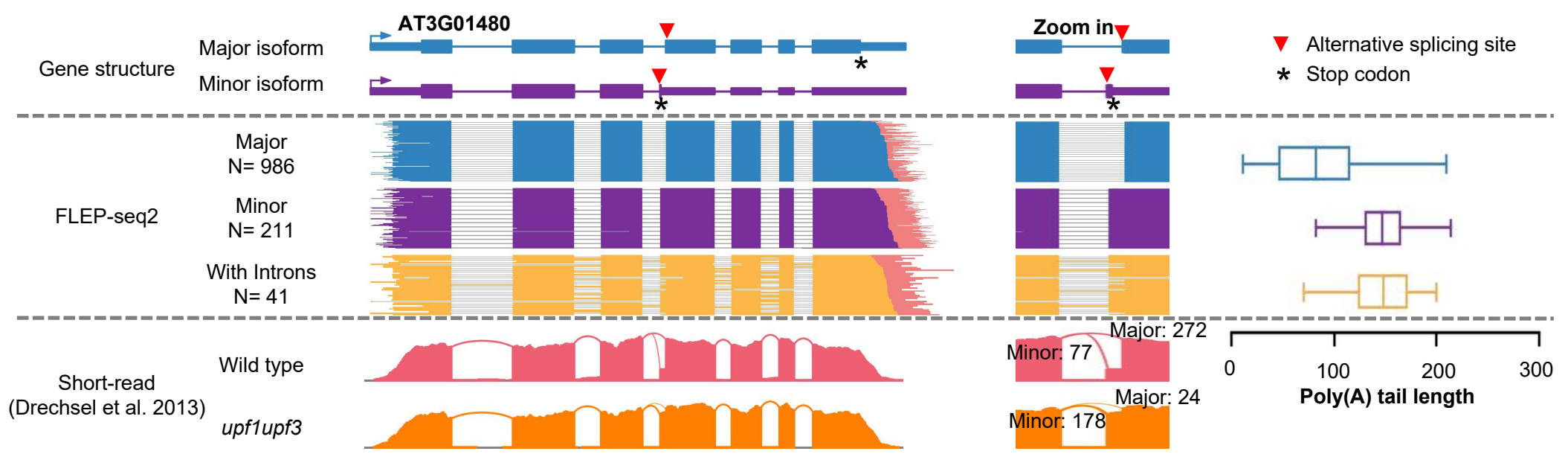

Supplemental Figure 5. Example of alternative splicing isoforms showing differential poly(A) tail lengths. 


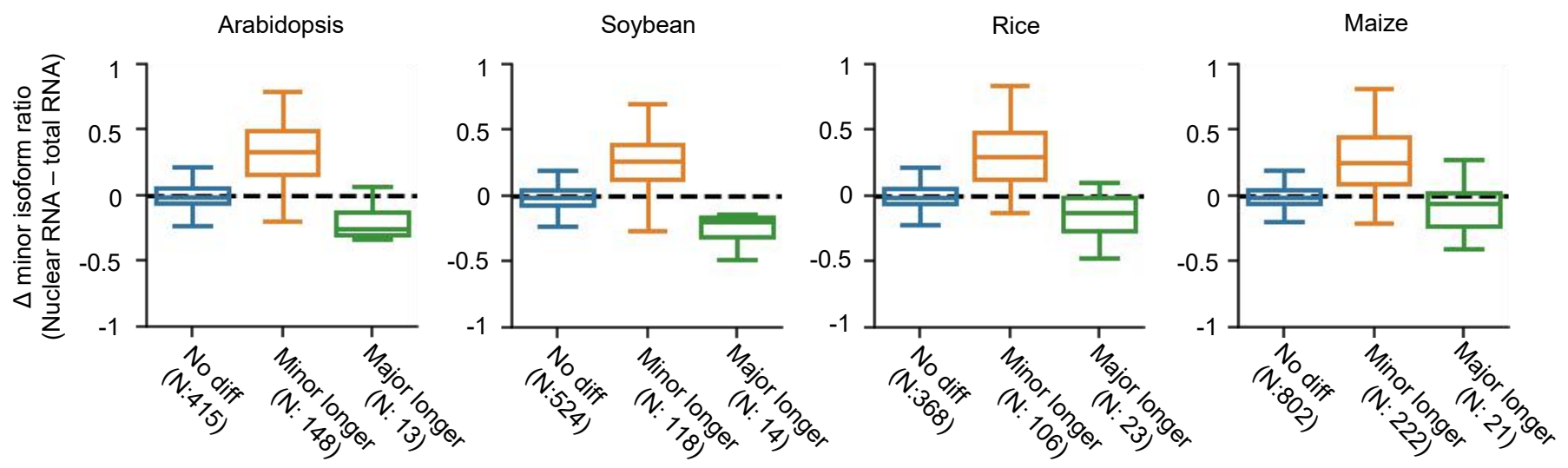

Supplemental Figure 6. Boxplot showing the distribution of $\Delta$ minor isoform ratio (minor/[minor+major]) between nuclear RNAs and total RNAs. The sums of the number of minor and major isoform reads in both nuclear and total RNA samples are required to be more than 10. 


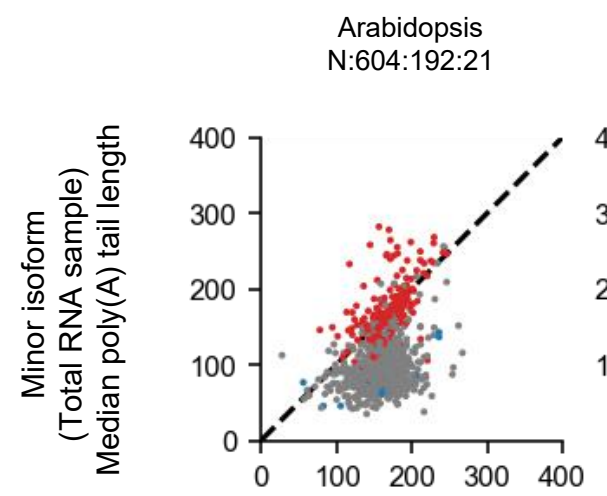

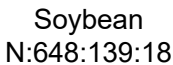

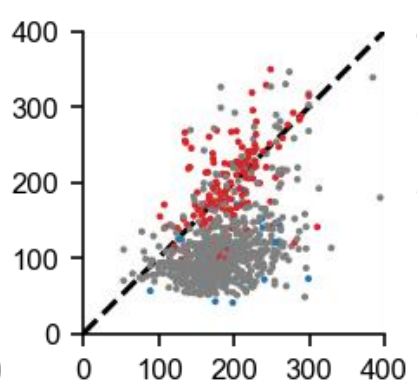

Rice

$N: 473: 139: 21$

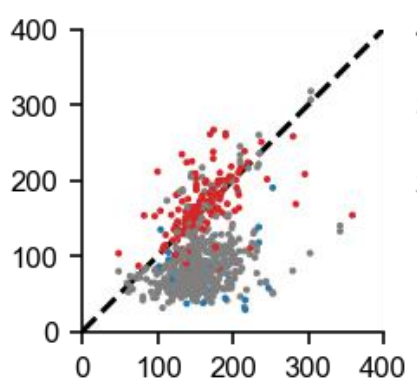

Maize

$\mathrm{N}: 1334: 317: 34$

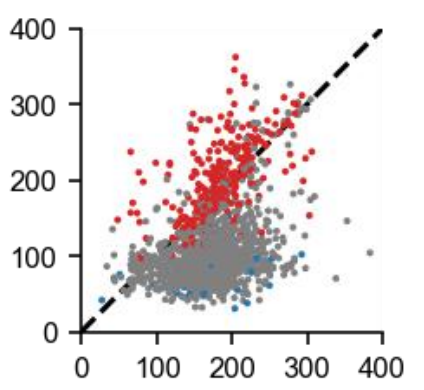

$N: \bullet: \bullet: \bullet$

- No diff

- Minor longer

- Major longer
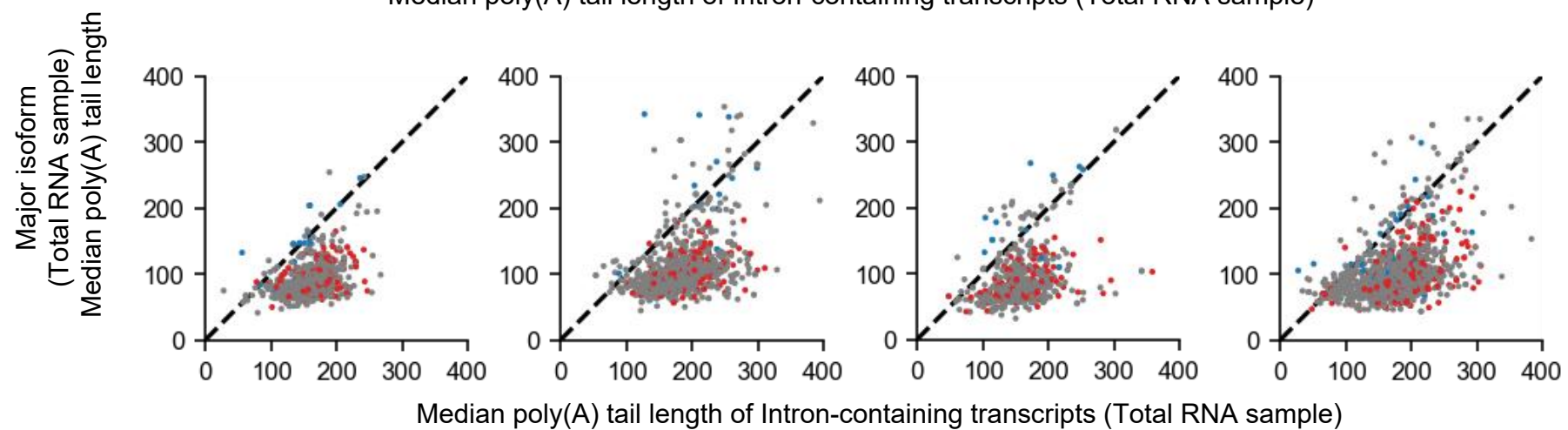

Supplemental Figure 7. The poly(A) tail lengths of minor isoforms with longer tail than major isoforms are highly consistent with the poly(A) tail of intron-containing transcripts. The comparison of median poly $(A)$ tail lengths between intron-containing transcripts with minor isoforms (upper) or major isoforms (below) in total RNA samples of different species. Only genes with at least 20 minor isoform reads, 20 major isoform reads and 20 intron-containing reads were used. The numbers of isoforms showing no differential, longer, and shorter poly $(A)$ tails were separated with ":" and labeled above each figure. 
bioRxiv preprint doi: https://doi.org/10.1101/2022.01.21.477033; this version posted January 22, 2022. The copyright holder for this preprint (which was not certified by peer review) is the author/funder, who has granted bioRxiv a license to display the preprint in perpetuity. It is made a The half life of mRNA in seedling (Szabo et al., 2020) 4.0 International licen

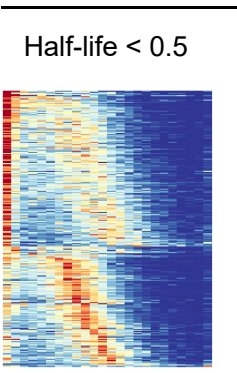

Shoot

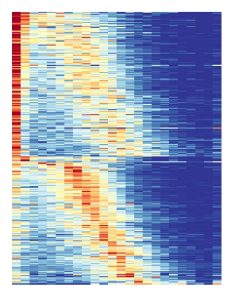

Root
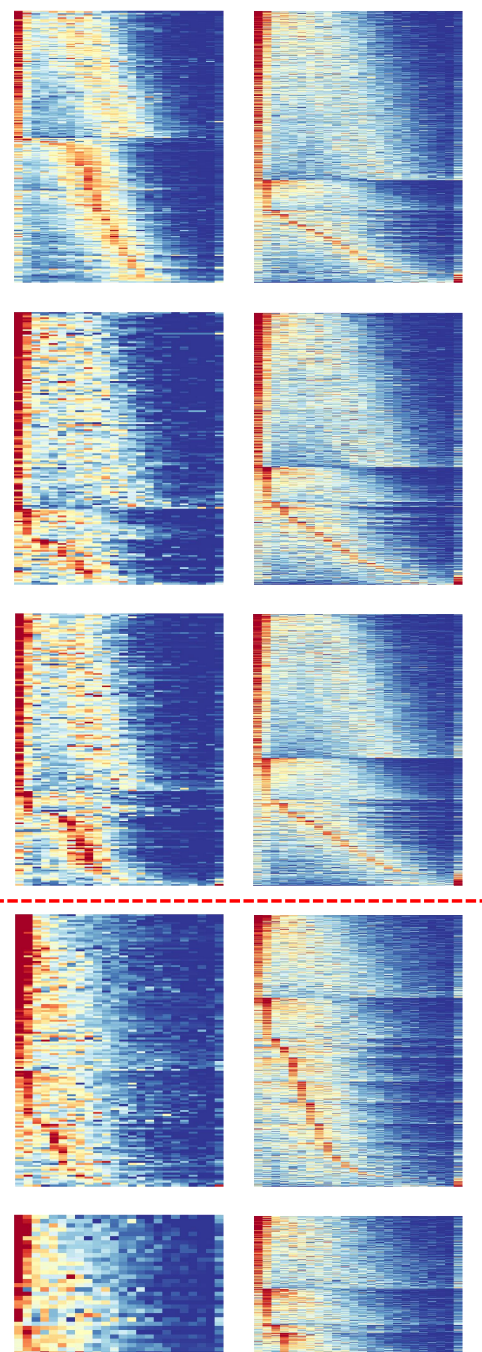

Pollen
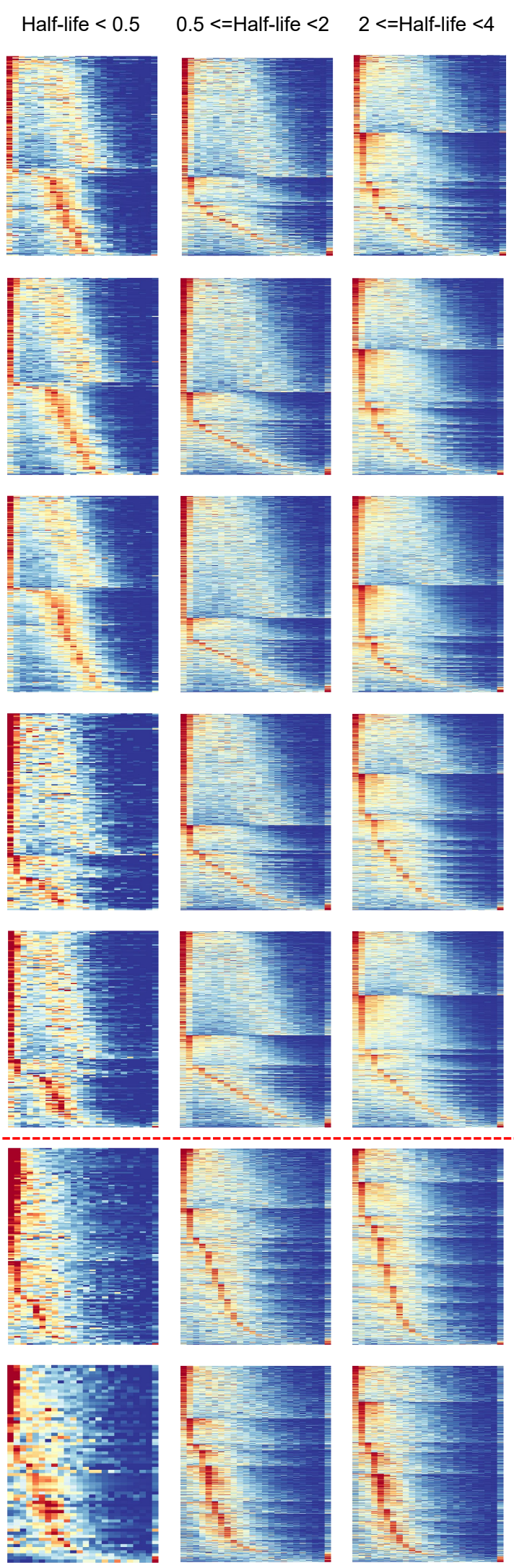

Half-life $>4$

Half-life $>4$ in

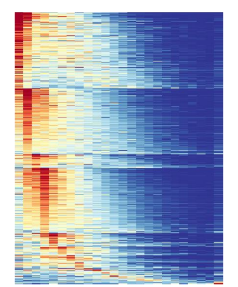

all three replicates
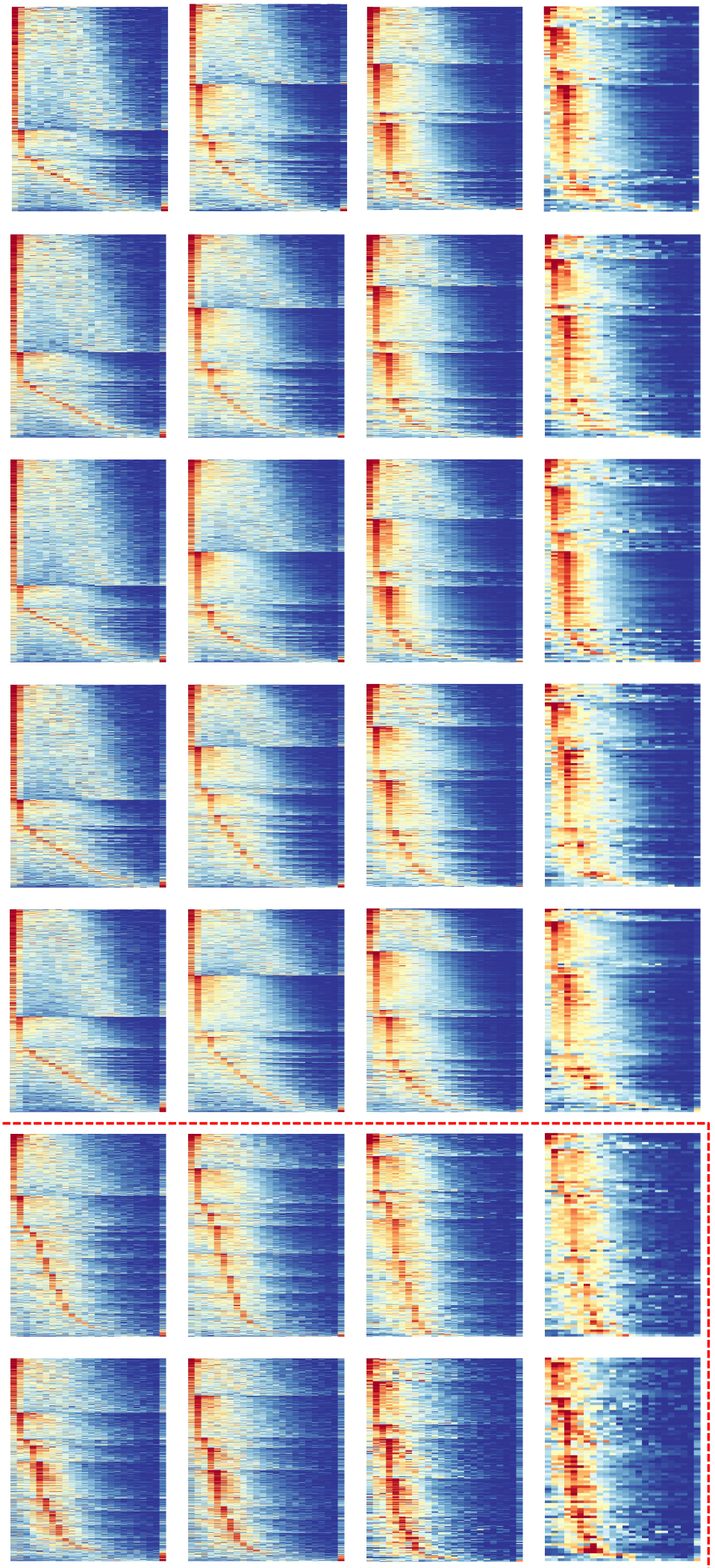

10501001502002501050100150200250105010015020025010501001502002501050100150200250 Poly(A) tail length (nt)

$$
\begin{gathered}
\text { Ratio } \\
\text { (10nt bin) }
\end{gathered}
$$
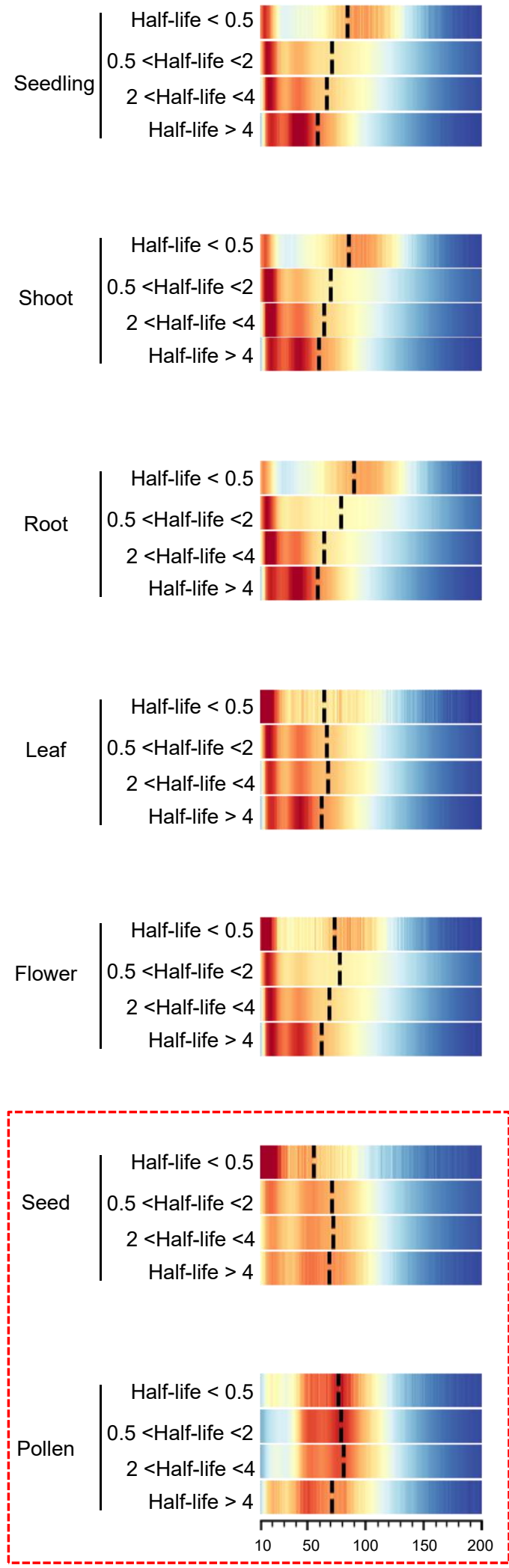

Poly(A) tail length (nt)

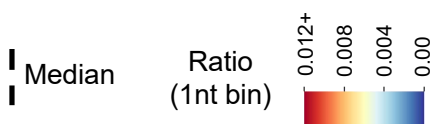

Supplemental Figure 8. The poly(A) tail lengths of transcripts with short half-lives in seedling are also enriched at 50-100 nt in pollen. a, Heatmap plot showing the poly $(A)$ tail length distribution of genes showing different half-lives. The mRNA half-life data of seedling was reported in previous paper (Szabo et al., $2020)$. Each row in the plot represent the poly(A) tail length distribution a one gene. Only genes with at least 50 reads were used. N: gene number. b, The bulk poly(A) tail length distribution of genes with different mRNA half-lives. 
bioRxiv preprint doi: https://doi.org/10.1101/2022.01.21.477033; this version posted January 22, 2022. The copyright holder for this preprint (which was not certified by peer review) is the author/funder, who has granted bioRxiv a license to display the preprint in perpetuity. It is made available under aCC-BY-NC-ND 4.0 International license.

a

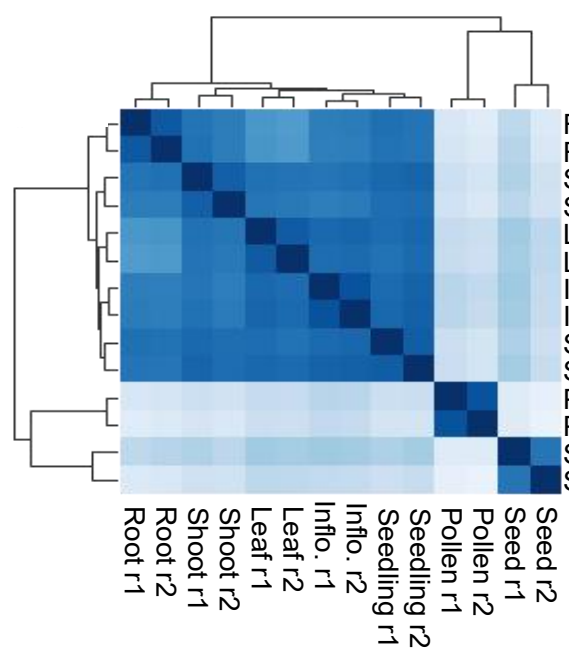

b
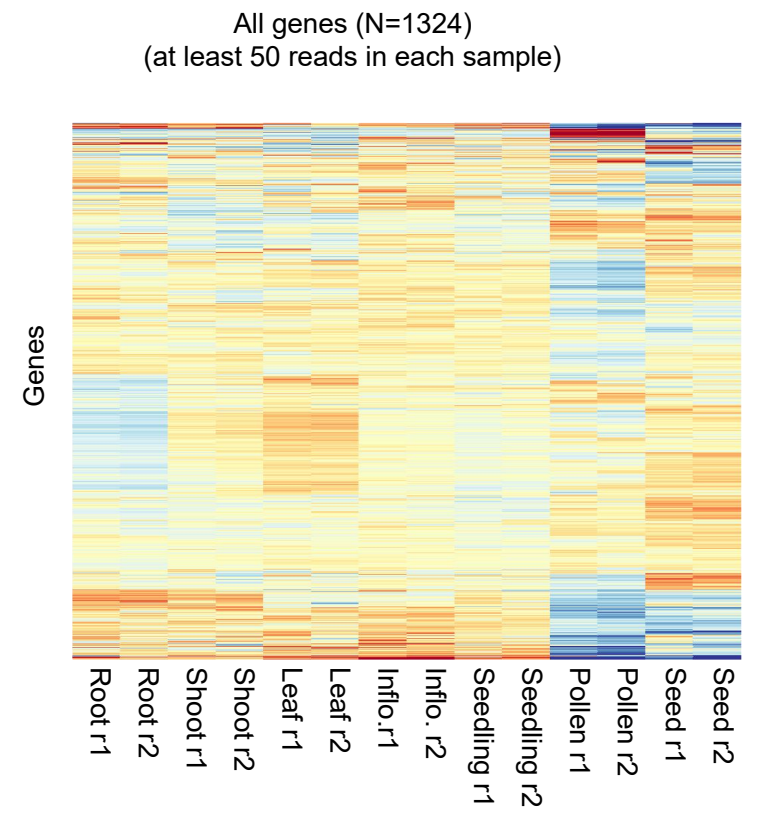

C

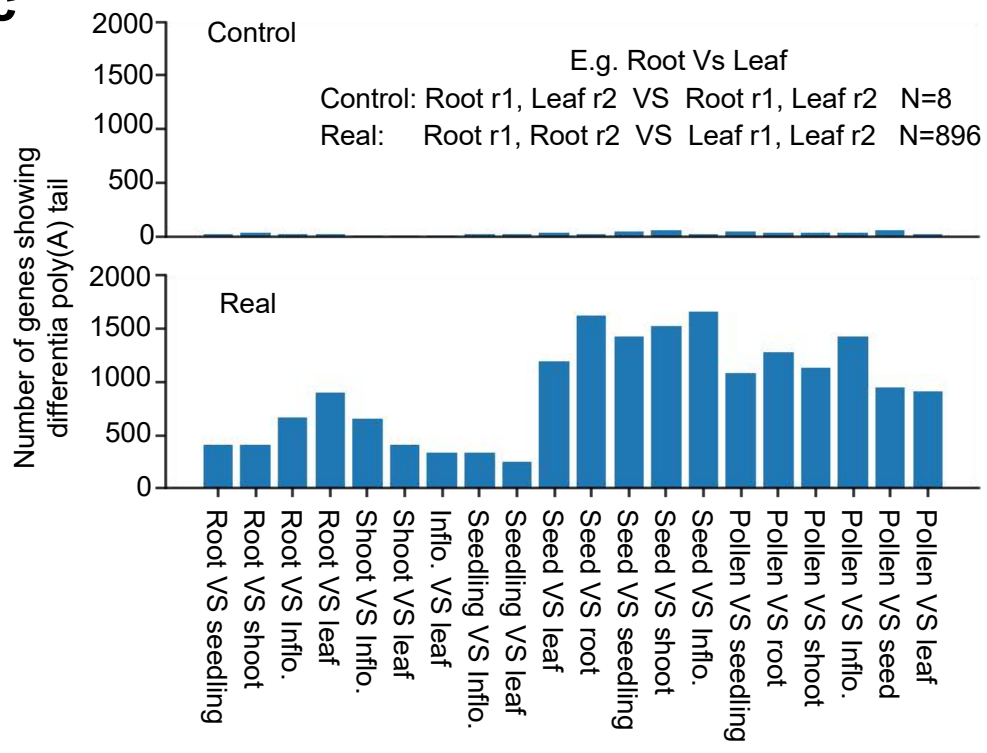

Genes showing significantly differentially regulated poly $(\mathrm{A})$ tails in at least one tissue pair $(\mathrm{N}=479)$ (at least 50 reads in each sample)

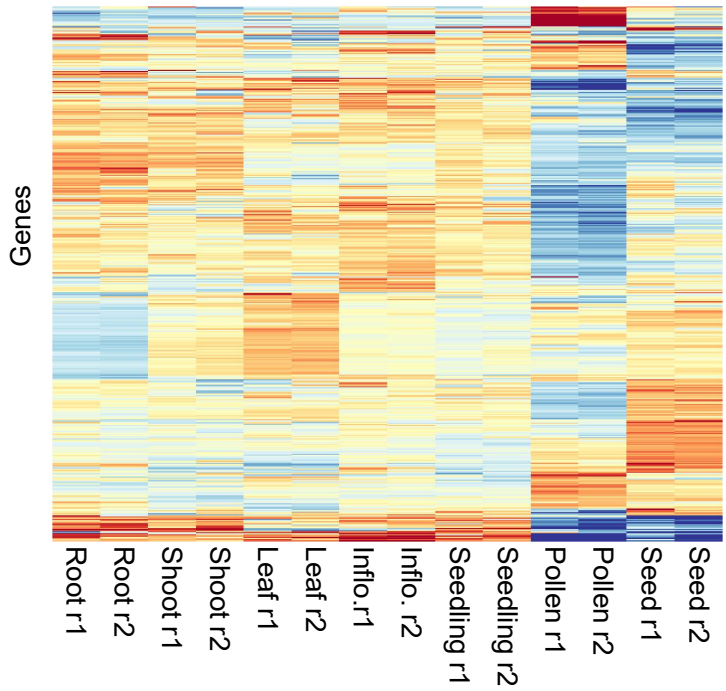

Relative Poly(A) tail length (Sample value mean value of all 14 samples)

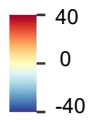

Supplemental Figure 9. Tissue-specific and evolutionarily-conserved regulation of poly(A) tail length in Arabidopsis. a, The median poly(A) tail length correlation matrix of different samples. b. Heatmap plot showing the relative median poly $(A)$ tail length of genes among different tissues. $\mathbf{c}$, The number of genes identified to show differential poly(A) tail length distribution in different tissues (Real, bottom panel) and in random data (Control, upper panel). Inflo.: Inflorescence. r1: biological replicate 1; r2: biological replicate 2 . 
bioRxiv preprint doi: https://doi.org/10.1101/2022.01.21.477033; this version posted January 22, 2022. The copyright holder for this preprint (which was not certified by peer review) is the author/funder, who has granted bioRxiv a license to display the preprint in perpetuity. It is made available under aCC-BY-NC-ND 4.0 International license.

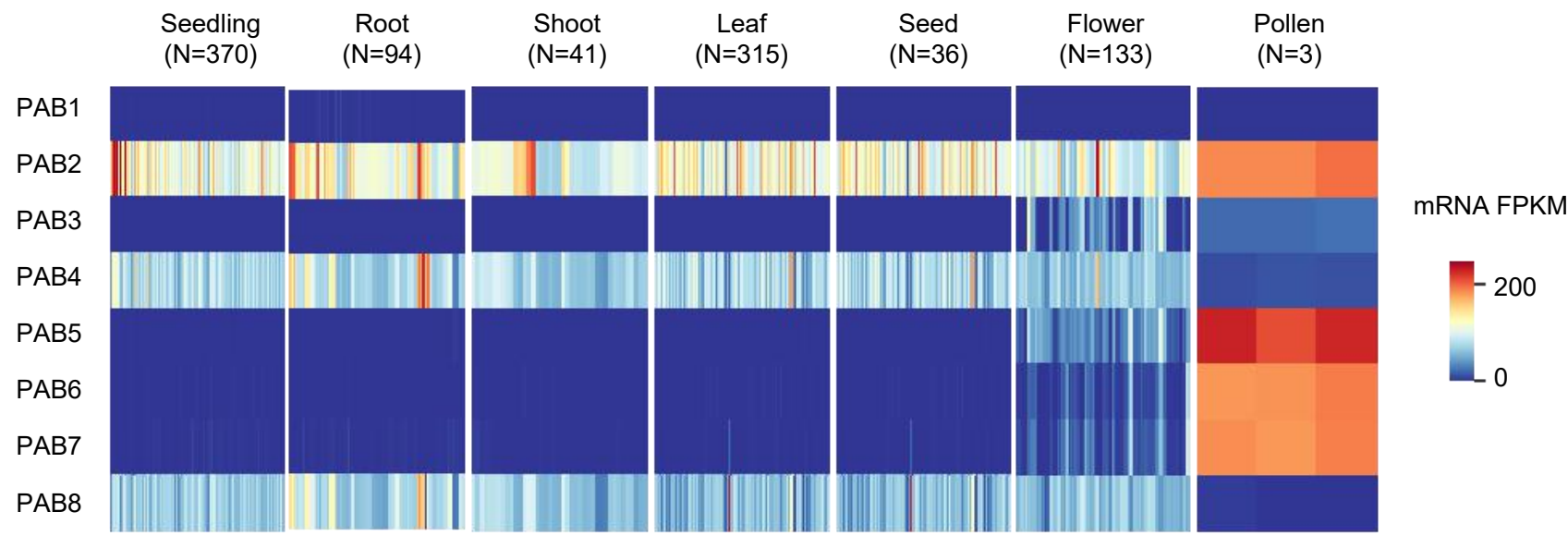

Supplemental Figure 10. Tissue-specific expression of Arabidopsis genes coding Poly(A) binding proteins (PAB). The gene expression values (Fragments per kilobase per million mapped fragments, FPKM value) of PABs in public RNA-seq data are downloaded from http://ipf.sustech.edu.cn/pub/athrna/. N: the number of RNA-seq libraries. 